\title{
Inner ideals and intrinsic subspaces in linear pair geometries
}

\author{
Wolfgang Bertram, Harald Löwe
}

\author{
Wolfgang Bertram \\ Université Henri Poincaré (Nancy I) - Institut Elie Cartan \\ B.P. 239 \\ F - 54506 Vandœuvre-lès-Nancy Cedex \\ e-mail: bertram@iecn.u-nancy.fr \\ Harald Löwe \\ Technische Universität Braunschweig - Institut Computational Mathematics \\ Pockelsstrasse 14 \\ D - 38106 Braunschweig \\ e-mail: h.loewe@tu-bs.de
}

\begin{abstract}
We introduce the notion of intrinsic subspaces of linear and affine pair geometries, which generalizes the one of projective subspaces of projective spaces. We prove that, when the affine pair geometry is the projective geometry of a Lie algebra introduced in [BeNe04], such intrinsic subspaces correspond to inner ideals in the associated Jordan pair, and we investigate the case of intrinsic subspaces defined by the Peirce-decomposition which is related to 5 -gradings of the projective Lie algebra. These examples, as well as the examples of general and Lagrangian flag geometries, lead to the conjecture that geometries of intrinsic subspaces tend to be themselves linear pair geometries.
\end{abstract}

\section{Contents:}

I. The axiomatic approach

1. Linear pair geometries

2. Intrinsic subspaces in linear pair geometries

II. The main examples

3. Flag geometries

4. Lagrangian flag geometries

5. Geometries of filtrations in Lie algebras

Appendix A: Inner ideals in matrix spaces

Appendix B: Inner ideals and Jordan theory

AMS-classification: $17 \mathrm{C} 37,17 \mathrm{C} 27,51 \mathrm{~N} 30,51 \mathrm{~B} 05$

Key words: linear and affine pair geometries, intrinsic subspace, inner ideal, Jordan pair, graded and filtered Lie algebras, flag geometries 


\section{Introduction}

The notion of subspace of a projective space is classical, and these subspaces have the nice property that, when looking at them from the affine point of view (i.e, after having removed some hyperplane at infinity) they just look like usual affine subspaces. Since projective spaces are generalized by Grassmannians (say, the Grassmannian $\mathcal{G r a s}_{p, q}(\mathbb{K})$ of $p$-spaces in some $p+q$ dimensional $\mathbb{K}$-vector space $W$ ), one may ask for a natural notion of subspace also in this and similar cases. One may expect, then, that the collection of all such subspaces forms a new kind of "geometry", with probably interesting properties and interacting with the theory of the Grassmannian geometry we started with. In this work, we will define such a kind of subspaces, called intrinsic subspaces, in a rather general framework which includes Grassmannians and many other examples (finite or infinite dimensional, or more generally in geometries over rings); we will give explicit constructions of such subspaces for many cases, and relate them to well-known objects that are called, in the theory of Jordan algebraic structures, inner ideals and which have attracted interest as possible material to build on them, e. g., quantum mechanical theories with a geometrical flavor (see, e.g., work of J.R. Faulkner [F80] and Chapter 9 of [BeNe05] for a link with the present work). Moreover, this approach permits to give a geometric definition of the notion of rank (or, using a terminology introduced by L. K. Hua [Hua45] for the classical matrix spaces, of arithmetic distance) in such spaces; the stratification of tangent spaces by the rank is very closely related to the generalized conformal structures introduced by S. Gindikin and S. Kaneyuki ([GiKa98]; cf. [Be00, Ch. IX]). In a sense, the present approach can be seen as a rather far-reaching generalization of these notions.

The basic idea is easily explained at the example of the Grassmannian geometry $\mathcal{X}=$ $\mathcal{G r a s}_{p, q}(\mathbb{K})$. Just like the projective space, the Grassmannian is covered by "affine parts", namely by the sets $\mathfrak{v}_{\alpha}$ of $p$-spaces that are complementary to some given $q$-space $\alpha$. Thus the affine parts are naturally parametrized by the "dual Grassmannian" $\mathcal{X}^{\prime}=\mathcal{G r a s}_{q, p}(\mathbb{K})$. Now, in the projective case (i.e., $p=1$ ) it is true that a subset which looks affinely with respect to one affinization looks affinely with respect to all affinizations. However, in the general case (i.e. $p>1$ and $q>1$ ) this is false! On the other hand, there exist some subsets which do have this property, and this defines precisely our intrinsic subspaces: an intrinsic subspace of $\mathcal{X}=\mathcal{G r a s}_{p, q}(\mathbb{K})$ is a subset $\mathcal{I} \subset \mathcal{X}$ such that all intersections $\mathcal{I}_{\alpha}=\mathcal{I} \cap \mathfrak{v}_{\alpha}$, where $\alpha$ runs through $\mathcal{X}^{\prime}$, are affine subspaces of $\mathfrak{v}_{\alpha}$.

The question arises then: how do such intrinsic subspaces look like, say, with respect to one fixed affinization $\mathfrak{v}=\mathfrak{v}_{o^{\prime}}$ ? Of course, they are linear (if they contain some fixed origin $o$ of $\mathfrak{v}$ ); but they must satisfy some additional condition. This additional condition is precisely the one of being an inner ideal of $\mathfrak{v}$. Let us recall, for readers not familiar with Jordan theory, that the notion of inner ideal is a simultaneous generalization of those of left and right ideals in an associative algebra. For instance, in the matrix algebra $A=M(n, n ; \mathbb{K})$ over a field $\mathbb{K}$, all left ideals are, with respect to a suitable basis, described as sets of matrices of the form $\left(\begin{array}{l}* 0 \\ * 0\end{array}\right)$; identifying $A$ with $\operatorname{End}_{\mathbb{K}}(U)$ where $U=\mathbb{K}^{n}$, we can write them as $I_{E}=\{f \in A \mid E \subset \operatorname{ker} f\}$ for some fixed subspace $E \subset U$. Simarly, right ideals are of the form $\left(\begin{array}{c}* * \\ 0\end{array}\right)$, resp. $J_{F}=\{f \in A \mid \operatorname{im} f \subset F\}$ for a subspace $F \subset U$. An inner ideal in $A=M(n, n ; \mathbb{K})$ is a subspace $I$ which is stable under multiplication from the inside in the trilinear product

$$
T: A \times A \times A \rightarrow A, \quad(f, g, h) \mapsto f g h+h g f,
$$

i.e., it satisfies the condition $T(I, A, I) \subset I$. It is immediate that all left or right ideals of $A$ are inner, and that an arbitrary intersection of inner ideals is again an inner ideal. Hence 
intersections of left- and right associative ideals are again inner ideals. These intersections are matrix spaces of the form $\left(\begin{array}{ll}* & 0 \\ 0 & 0\end{array}\right)$; more precisely, they are described as

$$
I_{E, F}:=\{f \in \operatorname{End}(U) \mid \operatorname{ker}(f) \subset E, \operatorname{im}(f) \subset F\}
$$

The notion of inner ideal depends only on the symmetrized product $x \bullet y=\frac{x y+y x}{2}$ in the associative algebra $A$ and makes in fact sense for any Jordan algebraic structure (-algebra, triple system or -pair). For instance, the algebra of matrices may be replaced by two spaces of rectangular matrices,

$$
V^{+}=M(p, q ; \mathbb{K})=\operatorname{Hom}_{\mathbb{K}}(U, W), \quad V^{-}=M(q, p ; \mathbb{K})=\operatorname{Hom}_{\mathbb{K}}(W, U),
$$

where the trilinear products $T^{ \pm}: V^{ \pm} \times V^{\mp} \times V^{ \pm} \rightarrow V^{ \pm} \times$and inner ideals (in $V^{+}$) are defined by the same formulae as above. Now, coming back to the Grassmannian example, the affine part $\mathfrak{v}$ is naturally identified with the space $V^{+}$, and we show then (Theorem 3.11) that the affine pictures $I=\mathfrak{v} \cap \mathcal{I}$ of intrinsic subspaces $\mathcal{I}$ are precisely the inner ideals of $V^{+}$. In this case, the inner ideals can be classified: they are all of the form $I_{E, F}$ as defined above (Proposition A.4). Therefore the spaces $I_{E, F}$ are precisely the affine pictures of intrinsic subspaces. For the case of the matrix spaces, we have gathered the basic facts on inner ideals in the first appendix (Appendix A), which uses only elementary linear algebra. The general Jordan theoretic notions are given in the second appendix (Appendix B), which is needed only for Chapter 5 . We hope that in this way the present work becomes accessible to readers who are interested in geometry but are not necessarily specialists in Jordan theory.

The general framework in which we can define intrinsic subspaces, called linear pair geometries, is defined in Chapter 1: it is given by a pair $\left(\mathcal{X}^{+}, \mathcal{X}^{-}\right)$of sets (we think of $\mathcal{X}^{-}$as the "dual" of $\mathcal{X}^{+}$and therefore write also $\left(\mathcal{X}, \mathcal{X}^{\prime}\right)$ ), together with a binary relation $T$ on $\mathcal{X}^{+} \times \mathcal{X}^{-}$, called transversality or remoteness (in the Grassmann case, $x \top \alpha$ means that $\alpha$ is a complementary subspace of $x$ ) such that for every $x \in \mathcal{X}^{+}$there is $\alpha \in \mathcal{X}^{-}$with $x \top \alpha$, and vice versa; then we assume that, for any transversal pair $(y, \alpha)$, the set $\mathfrak{v}_{\alpha}:=\left\{x \in \mathcal{X}^{+} \mid x \top \alpha\right\}$, with origin $y$, is equipped with a linear (i.e. $\mathbb{K}$-module) structure, and vice versa. As mentioned above, this is true in the Grassmannian case; but in that case, moreover, the underlying affine structure of $\mathfrak{v}_{\alpha}$ does not depend on $x$; we then say that the geometry is an affine pair geometry. Affine pair geometries have been introduced in [Be02], where it has been shown that a special class of such geometries, called generalized projective geometries, are essentially equivalent to Jordan algebraic structures. We prove in this work (Theorem 5.6) that, under this equivalence, intrinsic subspaces of the geometry correspond to inner ideals of the Jordan structure.

Let us now comment on the question whether the collection of all intrinsic subspaces forms some interesting kind of geometry. Although at present we do not have a general theory allowing to understand these geometries, the examples indicate that this is indeed the case: namely, it seems that geometries of intrinsic subspaces in linear pair geometries tend to be themselves again linear pair geometries, but in general these geometries are no longer affine pair geometries, even if the geometry we started with was one. In fact, this is our main motivation to work, from the beginning on, in this bigger class of geometries. For instance, if we start with a Grassmann geometry $\left(\mathcal{X}, \mathcal{X}^{\prime}\right)=\left(\mathcal{G r a s}_{p, q}(\mathbb{K}), \mathcal{G} \operatorname{ras}_{q, p}(\mathbb{K})\right)$ (which is an affine pair geometry), then the result stated above can be rephrased by saying that intrinsic subspaces are parametrized by "short flags" $0 \subset \mathfrak{h} \subset \mathfrak{k} \subset \mathbb{K}^{p+q}$, subject to the condition $\operatorname{dim} \mathfrak{h} \leq p \leq \operatorname{dim} \mathfrak{k}$ : to such a flag corresponds the intrinsic subspace

$$
\mathcal{I}_{\mathfrak{h}, \mathfrak{k}}=\{x \in \mathcal{X} \mid \mathfrak{h} \subset x \subset \mathfrak{k}\}
$$

(such subspaces of Grassmannians are also called Grassmann lines, cf. [Bue95, p. 49]). Thus the geometry of intrinsic subspaces corresponds, in this case, to flag varieties of short flags. We show 
in Chapter 3 that flag varieties of general linear groups (and, similarly, flag varieties of orthogonal and symplectic groups, see Chapter 4 ) are indeed examples of linear, non affine pair geometries (Theorem 3.5) and we construct standard examples of intrinsic subspaces for such geometries (Theorem 3.8). Similar observations can be made for the case of the projective geometry of a Lie algebra, i.e., for the affine pair geometry of inner 3 -filtrations of a Lie algebra $\mathfrak{g}$ (defined in [BeNe04]): in this case, intrinsic subspaces correspond exactly to inner ideals in the associated Jordan pair (Theorem 5.6), and the important class of intrinsic subspaces corresponding to inner ideals defined by Peirce-decompositions is closely related to 5 -gradings and 5 -filtrations of $\mathfrak{g}$ (Theorem 5.8). The geometry of 5 -gradings of a Lie algebra is not yet fully understood; we conjecture that it is also a linear pair geometry. Thus, at this point, several problems and topics for further research arise naturally:

(1) What kinds of graded Lie algebras give rise to linear pair geometries? For 3-graded Lie algebras, a result of this type has been proved in [BeNe04] (see the summary in Section 5); is an analogue true for other kinds of gradings?

(2) Do linear pair geometries of the kind mentioned in (1) correspond to some kind of "noncommutative Jordan structures" in a similar way as generalized projective geometries correspond to usual (commutative) Jordan structures?

(3) If the answer to (1) or (2) is positive, then it seems possible to develop the theory of these geometries, similarly as for the Jordan case in [BeNe04] and [BeNe05], in arbitrary dimension and over general base fields and rings, in particular furnishing interesting classes of infinite dimensional manifolds.

(4) Finally, if it is true that geometries of intrinsic subspaces tend to be linear pair geometries themselves, one may ask for a conceptual proof of this fact. As a first step, it seems to be important to find a good geometric analog of the complementation of inner ideals in Jordan pairs (see [LoNe94]) in order to define a structure of pair geometry on the geometry of intrinsic subspaces.

Notation and terminology. Throughout the paper, let $\mathbb{K}$ denote a ring with unit 1 such that 2 is invertible in $\mathbb{K}$. In some parts of the text, we will assume that $\mathbb{K}$ is a field. We also use the terms linear (sub)space instead of $\mathbb{K}-($ sub)module and try to use systematically gothic letters $\mathfrak{v}, \mathfrak{o}, \mathfrak{e}, \mathfrak{f}, \ldots$ for linear spaces (or flags of linear spaces) and calligraphic letters $\mathcal{X}, \mathcal{I}, \mathcal{F}, \mathcal{L}, \ldots$ for non-linear objects.

Acknowledgements. The second author thanks the Institut Elie Cartan for hospitality and support during his research visit in 2003, and the first author thanks the Mathematical Institute of the TU Braunschweig for hospality during his visit in 2004.

\section{Linear pair geometries}

1.1. Pair geometries. A pair geometry is given by a pair of sets $\left(\mathcal{X}, \mathcal{X}^{\prime}\right)$, together with a subset $\left(\mathcal{X} \times \mathcal{X}^{\prime}\right)^{\top} \subset \mathcal{X} \times \mathcal{X}^{\prime}$, such that the following conditions are satisfied:

- for all $\alpha \in \mathcal{X}^{\prime}$ there exists $x \in \mathcal{X}$ such that $(x, \alpha) \in\left(\mathcal{X} \times \mathcal{X}^{\prime}\right)^{\top}$, and

- for all $x \in \mathcal{X}$, there exists $\alpha \in \mathcal{X}^{\prime}$ such that $(x, \alpha) \in\left(\mathcal{X} \times \mathcal{X}^{\prime}\right)^{\top}$.

If $(x, \alpha)$ belongs to $\left(\mathcal{X} \times \mathcal{X}^{\prime}\right)^{\top}$, then we shall write $x \top \alpha$ and call the pair $(x, \alpha)$ is transversal (according to the context, we may also use the terminology remote, distant or in general position). Notice that the conditions stated above simply express that $\mathcal{X}$ is covered by the sets

$$
\mathfrak{v}_{\alpha}:=\alpha^{\top}:=\{x \in \mathcal{X} \mid x \top \alpha\}
$$


for $\alpha \in \mathcal{X}^{\prime}$, and $\mathcal{X}^{\prime}$ is covered by the sets $\mathfrak{v}_{x}^{\prime}$ defined dually for $x \in \mathcal{X}$.

1.2. Linear and affine pair geometries. Let $\mathbb{K}$ be a commutative unital ring. A linear pair geometry over $\mathbb{K}$ is a pair geometry such that, for every transversal pair $x \top \alpha$, both $\left(\mathfrak{v}_{\alpha}, x\right)$ and $\left(\mathfrak{v}_{x}^{\prime}, \alpha\right)$ are equipped with a $\mathbb{K}$-module structure (with zero vectors $x$ and $\alpha$, respectively). We adress the sets $\mathfrak{v}_{a}, a \in \mathcal{X}^{\prime}$, as linear parts or linear charts of $\mathcal{X}$, and dually for $\mathcal{X}^{\prime}$. We say that a linear pair geometry is of commutative type, or shorter: is an affine pair geometry, if, for all $a \in \mathcal{X}^{\prime}$, the underlying affine structure of the $\mathbb{K}$-modules $\left(\mathfrak{v}_{a}, x\right)$ does not depend on the choice of the element $x \in a^{\top}$, and dually. Thus we may speak of "the" affine space $\mathfrak{v}_{a}$, and the linear structures on $\mathfrak{v}_{a}$ are related among each other by the usual formulae

$$
u+{ }_{x} v=u-x+v, \quad r_{x} v=(1-r) x+r v
$$

where addition and scalar multiplication without subscript refers to one fixed origin $0 \in \mathfrak{v}_{a}$.

1.3. The structure maps. Let $\left(\mathcal{X}, \mathcal{X}^{\prime}\right)$ be a linear pair geometry. If $(x, a),(y, a) \in\left(\mathcal{X} \times \mathcal{X}^{\prime}\right)^{\top}$ and $r \in \mathbb{K}$, then let $r_{x, a}(y):=r y$ denote the product $r \cdot y$ in the $\mathbb{K}$-module $\mathfrak{v}_{a}$ with zero vector $x$. Moreover, we define the structure maps by

$$
\Pi_{r}:\left(\mathcal{X} \times \mathcal{X}^{\prime} \times \mathcal{X}\right)^{\top} \rightarrow \mathcal{X}, \quad(x, a, y) \mapsto \Pi_{r}(x, a, y):=r_{x, a}(y),
$$

where

$$
\left(\mathcal{X} \times \mathcal{X}^{\prime} \times \mathcal{X}\right)^{\top}=\left\{(x, a, y) \in \mathcal{X} \times \mathcal{X}^{\prime} \times \mathcal{X} \mid x \top a, y \top a\right\}
$$

and dually the maps $\boldsymbol{\Pi}_{r}^{\prime}$ are defined. Similarly, we derive structure maps from the vector addition:

$$
\boldsymbol{\Sigma}:\left(\mathcal{X} \times \mathcal{X}^{\prime} \times \mathcal{X} \times \mathcal{X}\right)^{\top} \rightarrow \mathcal{X}, \quad(x, a, y, z) \mapsto \boldsymbol{\Sigma}(x, a, y, z):=y+_{x, a} z,
$$

where the sum is taken in the $\mathbb{K}$-module $\left(\mathfrak{v}_{a}, x\right)$ and where the set $\left(\mathcal{X} \times \mathcal{X}^{\prime} \times \mathcal{X} \times \mathcal{X}\right)^{\top}$ is defined by a similar condition as in (1.2). Dually, $\boldsymbol{\Sigma}^{\prime}$ is defined.

1.4. Duality. The linear pair geometry $\left(\mathcal{X}^{\prime}, \mathcal{X} ; \top ; \Pi^{\prime}, \boldsymbol{\Pi}, \boldsymbol{\Sigma}^{\prime}, \boldsymbol{\Sigma}\right)$ is called the dual geometry of $\left(\mathcal{X}, \mathcal{X}^{\prime} ; \top ; \Pi, \Pi^{\prime}, \boldsymbol{\Sigma}, \boldsymbol{\Sigma}^{\prime}\right)$.

1.5. Morphisms. I. Homomorphisms of linear pair geometries are pairs of maps $\left(g, g^{\prime}\right)$ : $\left(\mathcal{X}, \mathcal{X}^{\prime}\right) \rightarrow\left(\mathcal{Y}, \mathcal{Y}^{\prime}\right)$ which preserve transversality and which are compatible with the multiplication and addition maps in the sense that $g \boldsymbol{\Pi}_{r}(x, a, y)=\boldsymbol{\Pi}_{r}\left(g x, g^{\prime} a, g y\right)$, etc. This means simply that $g$ induces by restriction a linear map from $\left(\mathfrak{v}_{a}, x\right)$ to $\left(\mathfrak{v}_{g^{\prime}} a, g(x)\right)$, and dually. In particular, we can speak of the automorphism group $\operatorname{Aut}\left(\mathcal{X}, \mathcal{X}^{\prime}\right)$ of a linear pair geometry $\left(\mathcal{X}, \mathcal{X}^{\prime}\right)$. An anti-automorphism is an isomorphism of $\left(\mathcal{X}, \mathcal{X}^{\prime}\right)$ onto its dual geometry; a correlation is an anti-automorphism of order 2 (i.e. $g^{\prime}=g^{-1}$ ); a correlation is called a polarity if it admits non-isotropic points (i.e. there is $x \in \mathcal{X}$ such that $x \top g(x))$ and a null-system if all points are isotropic.

A base point in $\left(\mathcal{X}, \mathcal{X}^{\prime}\right)$ is a pair $\left(o, o^{\prime}\right) \in\left(\mathcal{X} \times \mathcal{X}^{\prime}\right)^{\top}$. The structure group is the group $\operatorname{Aut}\left(X, X^{\prime} ; o, o^{\prime}\right)$ of automorphisms fixing the base point. ¿From the definitions it follows that this group acts linearly on $\mathfrak{v}_{o^{\prime}} \times \mathfrak{v}_{o}^{\prime}$.

1.6. Morphisms. II. Adjoint pairs of morphisms are given by pairs $g: \mathcal{X} \rightarrow \mathcal{Y}, h: \mathcal{Y}^{\prime} \rightarrow \mathcal{X}^{\prime}$ such that transversality is preserved in the sense that $x \top h(a)$ iff $g(x) \top a$, and, whenever $(x, h(a))$ and $(y, h(a))$ are transversal, then

$$
g \boldsymbol{\Pi}_{r}(x, h a, y)=\Pi_{r}(g x, a, g y)
$$

and similarly for $\boldsymbol{\Pi}_{r}^{\prime}, \boldsymbol{\Sigma}$ and $\boldsymbol{\Sigma}^{\prime}$; we then write $h=g^{t}$. This means that $g$ induces a linear map from $\left(\mathfrak{v}_{h a}, x\right)$ to $\left(\mathfrak{v}_{a}, g x\right)$. Note that every isomorphism $\left(g, g^{\prime}\right)$ gives rise to an adjoint pair $\left(g,\left(g^{\prime}\right)^{-1}\right)$, and conversely, every bijective adjoint pair gives rise to an isomorphism. 
1.7. Connectedness. We will say that two points $x, y \in \mathcal{X}$ are on a common chart if there is $a \in \mathcal{X}^{\prime}$, such that $x, y \in \mathfrak{v}_{a}$. Equivalently, $\mathfrak{v}_{x}^{\prime} \cap \mathfrak{v}_{y}^{\prime} \neq \varnothing$. We will say that $x, y \in \mathcal{X}$ are connected if there is a sequence of points $x_{0}=x, x_{1}, \ldots, x_{k}=y$ such that $x_{i}$ and $x_{i+1}$ are on a common chart. This defines an equivalence relation on $\mathcal{X}$ whose equivalence classes are called connected components of $\mathcal{X}$. By duality, connected components of $\mathcal{X}^{\prime}$ are also defined. The geometry is called connected if both $\mathcal{X}$ and $\mathcal{X}^{\prime}$ are connected.

1.8. Stability. The pair geometry $\left(\mathcal{X}, \mathcal{X}^{\prime}, \top\right)$ will be called stable if any two points $x, y \in \mathcal{X}$ are on a common chart, and dually for any points $a, b \in \mathcal{X}^{\prime}$. Clearly, a stable geometry is connected (the converse is not true).

1.9. Direct products. The direct product of two linear pair geometries, or of a family of linear pair geometries, is defined in the obvious way.

1.10. Non-degeneracy. The geometry is called non-degenerate if, for $a, b \in \mathcal{X}^{\prime}, \mathfrak{v}_{a}=\mathfrak{v}_{b}$ implies $a=b$, and dually.

1.11. Flat geometries. For any $\mathbb{K}$-module $V$ we may consider the geometry $\left(\mathcal{X}, \mathcal{X}^{\prime}\right)=(V, p)$, where $\mathcal{X}^{\prime}=\{p\}$ is reduced to a point, and the multiplication map $\Pi_{r}(x, p, y)=(1-r) x+r y$ reflects just the usual affine structure of $V$. In a similar way, we may define the flat geometry $\left(\mathcal{X}, \mathcal{X}^{\prime}\right)=\left(V, V^{\prime}\right)$ associated to a pair of $\mathbb{K}$-modules by letting the multiplication maps be independent of the middle argument. These geometries are the most degenerate cases.

\section{Intrinsic subspaces in linear pair geometries}

2.1. Subspaces. Assume $\left(\mathcal{X}, \mathcal{X}^{\prime}\right)$ is a linear pair geometry over $\mathbb{K}$. A pair $\left(\mathcal{Y}, \mathcal{Y}^{\prime}\right)$ of subsets $\mathcal{Y} \subset \mathcal{X}, \mathcal{Y}^{\prime} \subset \mathcal{X}^{\prime}$ is called a subspace of $\left(\mathcal{X}, \mathcal{X}^{\prime}\right)$, if:

(1) For all $x \in \mathcal{Y}$ there exists an element $a \in \mathcal{Y}^{\prime}$ such that $x \top a$. Dually, for all $a \in \mathcal{Y}^{\prime}$ there exists an element $x \in \mathcal{Y}$ such that $x \top a$.

(2) For every $(x, a) \in\left(\mathcal{Y} \times \mathcal{Y}^{\prime}\right)^{\top}$ the set $\mathcal{Y}_{a}:=\mathcal{Y} \cap \mathfrak{v}_{a}$ is a linear subspace of $\left(\mathfrak{v}_{a}, x\right)$ and $\mathcal{Y}_{x}^{\prime}:=\mathcal{Y}^{\prime} \cap \mathfrak{v}_{x}^{\prime}$ is a linear subspace of $\left(\mathfrak{v}_{x}^{\prime}, a\right)$.

Then clearly $\left(\mathcal{Y}, \mathcal{Y}^{\prime}\right)$ is a linear pair geometry in its own right. Examples are provided by Lagrangian geometries, which are subspaces of Grassmann geometries, cp. Section 4.

2.2. Intrinsic subspaces. A subset $\mathcal{I} \subset \mathcal{X}$ is called an intrinsic subspace (in $\mathcal{X}$ ), if $\mathcal{I}$ appears linearly with respect to all possible linearizations or, more precisely, if for all $a \in \mathcal{X}^{\prime}$ with $\mathfrak{v}_{a} \cap \mathcal{I} \neq \varnothing$ and for all $x \in \mathfrak{v}_{a} \cap \mathcal{I}$, the set $\left(\mathfrak{v}_{a} \cap \mathcal{I}, x\right)$ is a linear subspace of $\left(\mathfrak{v}_{a}, x\right)$. Given an intrinsic subspace $\mathcal{I}$, we put

$$
\mathcal{I}^{\prime}:=\bigcup_{x \in \mathcal{I}} \mathfrak{v}_{x}^{\prime}
$$

Then $\left(\mathcal{I}, \mathcal{I}^{\prime}\right)$ is a subspace (in the sense of 2.1) having the particular property that $\mathcal{I}^{\prime}$ contains the whole linear part $\mathfrak{v}_{x}^{\prime}$ for every $x \in \mathcal{I}$.

Lemma 2.3. The intersection $\mathcal{I}:=\bigcap_{i \in J} \mathcal{Y}_{i}$ of an arbitrary family $\left(\mathcal{I}_{i}\right)_{i \in J}$ of intrinsic subspaces is an intrinsic subsace again.

Proof. Let $x \in \mathcal{I}$ and $a \in \mathfrak{v}_{x}$. If $i \in J$, then $\left(\mathcal{I}_{i} \cap \mathfrak{v}_{a}, x\right)$ is a linear subspace of $\left(\mathfrak{v}_{a}, x\right)$ and, hence, $\mathcal{I} \cap \mathfrak{v}_{x}=\cap_{i \in J}\left(\mathcal{I}_{i} \cap \mathfrak{v}_{x}\right)$ is a linear subspace of $\left(\mathfrak{v}_{a}, x\right)$, too. 
2.4. Principal intrinsic subspaces. Every singleton $\{x\}$ (with $x \in \mathcal{X}$ ) is an intrinsic subspace of $\mathcal{X}$. It is the smallest intrinsic subspace in $\mathcal{X}$ containing $x$. For $S \subset \mathcal{X}$, the subset

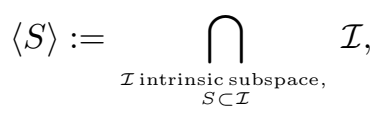

called the intrinsic subspace generated by $S$, is an intrinsic subspace, thanks to the preceding lemma. Clearly, $\langle S\rangle$ is the smallest intrinsic subspace containing $S$. For $x, y \in \mathcal{X}$, we shall write

$$
x \vee y:=\langle\{x, y\}\rangle \subset X,
$$

called the principal intrinsic subspace generated by $x$ and $y$. If $x, y$ are not on a common chart (cf. 1.5), then $x \vee y=\{x, y\}$, and if $x, y$ are on a common chart $\mathfrak{v}_{a}$, then $x \vee y$ contains at least the affine lines $\left\{\boldsymbol{\Pi}_{r}(x, a, y) \mid r \in \mathbb{K}\right\}$ and $\left\{\boldsymbol{\Pi}_{r}(y, a, x) \mid r \in \mathbb{K}\right\}$ with respect to $\left(\mathfrak{v}_{a}, x\right)$ and $\left(\mathfrak{v}_{a}, y\right)$ joining $x$ and $y$ (which are the same sets if our geometry is an affine pair geometry).

We write also $\mathcal{I} \wedge \mathcal{J}$ for the intersection of two intrinsic subspaces $\mathcal{I}$ and $\mathcal{J}$ and $\mathcal{I} \vee \mathcal{J}:=$ $\langle\mathcal{I} \cup \mathcal{J}\rangle$ for the smallest intrinsic subspace containing $\mathcal{I} \cup \mathcal{J}$. Then the set of all intrinsic subspaces (resp. the set of all intrinsic subspaces in $\mathcal{X}$ containing a given point $o \in \mathcal{X}$ ) forms a complete lattice with respect to inclusion and the operations $\wedge$ and $\vee$.

2.5. Trivial intrinsic subspaces. We say that an intrinsic subspace $\mathcal{I}$ is trivial if, for all $x \in \mathcal{I}$ and $a \top x$ the underlying affine structure of the $\mathbb{K}$-module $\mathfrak{m}:=\left(\mathfrak{v}_{a} \cap \mathcal{I}, x\right)$ depends neither on the choice of $x \in \mathfrak{m}$ nor on the choice of $a$ with $\mathfrak{m} \subset \mathfrak{v}_{a}$.

2.6. Intrinsic subspaces given by images of adjoint pairs. Assume $(g, h):\left(\mathcal{Y}, \mathcal{X}^{\prime}\right) \rightarrow$ $\left(\mathcal{X}, \mathcal{Y}^{\prime}\right)$ is an adjoint pair of morphisms (cf. 1.6). Then the image $\operatorname{im}(g)$ is an intrinsic subspace in $\mathcal{X}$ : in fact, whenever $x, y \in \mathcal{Y}$ and $a \in \mathfrak{v}_{g x}^{\prime} \cap \mathfrak{v}_{g y}^{\prime}$, we have

$$
\boldsymbol{\Pi}_{r}(g(x), a, g(y))=g \boldsymbol{\Pi}_{r}(x, h(a), y) \in \operatorname{im}(g),
$$

and similarly for $\boldsymbol{\Sigma}$; hence $\operatorname{im}(g)$ is an intrinsic subspace.

2.7. Flags, chain conditions and rank. A flag of intrinsic subspaces is a chain

$$
\ldots \mathcal{I}_{-2} \subset \mathcal{I}_{-1} \subset \mathcal{I}_{0} \subset \mathcal{I}_{1} \subset \ldots
$$

of intrinsic subspaces in $\mathcal{X}$, and its length, possibly infinite, is the number of strict inclusions. If a point $o \in \mathcal{X}$ is fixed, a principal flag centered at $o$ is given by a flag

$$
o \vee x_{-2} \subset o \vee x_{-1} \subset \ldots \subset o \vee x
$$

of principal intrinsic subspaces one of whose generators is $o$ and which shall all be included in some intrinsic subspace of the form $o \vee x$. The supremum of all lengths of (principal) flags of finite length is called the (principal) rank of $\mathcal{X}$, and the supremum of all lengths of principal flags of finite length centered at $o$ and included in $o \vee x$ is called the (principal) rank. We say that the geometry $\left(\mathcal{X}, \mathcal{X}^{\prime}\right)$ satisfies the descending (ascending) chain condition on principal subspaces (dcc, resp. acc) if every descending (ascending) flag of principal subspaces becomes stationary.

2.8. Intrinsic lines. Elements of $\mathcal{X}$ are called points. A connected intrinsic subspace $\mathcal{I}$ in $\mathcal{X}$ is called an intrinsic line if it is not a singleton and if all intrinsic subspaces properly contained in $\mathcal{I}$ are singletons. We will also use the term minimal intrinsic subspace. If $x$ and $y$ are different points on a line $\mathcal{I}$, then $x \vee y=\mathcal{I}$. A line is called simple if it is not a trivial intrinsic subspace. Thus affine lines in flat geometries of vector spaces are not considered as simple. Note that, in 
general, intrinsic lines need not exist; they do exist if our geometry satisfies a descending chain condition.

2.9. A word of warning concerning the "horizon." For any $\alpha \in \mathcal{X}^{\prime}$, we define the horizon (of the linear part corresponding to $\alpha$ ) to be the set

$$
H_{\alpha}:=\mathcal{X} \backslash \mathfrak{v}_{\alpha} .
$$

In general $H_{\alpha}$ will not define an intrinsic subspace in $\mathcal{X}$, nor is it the first component of some subspace $\left(\mathcal{Y}, \mathcal{Y}^{\prime}\right)$, and for this reason we do not use the term "hyperplane at infinity" which the reader might be used to from projective geometry. In fact, one can show that essentially the only example where $H_{\alpha}$ is an intrinsic subspace is the example of ordinary projective geometry over a field, $\left(\mathcal{X}, \mathcal{X}^{\prime}\right)=\left(\mathcal{G r a s}_{1, n}(\mathbb{K}), \mathcal{G} \operatorname{ras}_{n, 1}(\mathbb{K})\right)$ (see below, Chapter 3). On the other hand, in the case of geometries associated to Jordan pairs (cf. Section 5), there exist non-trivial relations between the description of the horizon and the notion of rank.

\section{Flag geometries}

In this and the next two chapters we discuss the most important examples of linear pair geometries and their intrinsic subspaces.

3.1. Transversality of flags. Assume $\mathfrak{m}$ is a $\mathbb{K}$-module and $k \in \mathbb{N}$. A flag of length $k$, or $k$-filtration of $\mathfrak{m}$, is a sequence $\mathfrak{f}=\left(\mathfrak{f}_{1}, \mathfrak{f}_{2}, \ldots, \mathfrak{f}_{k-1}, \mathfrak{f}_{k}\right)$ of ascending linear subspaces

$$
0 \subset \mathfrak{f}_{1} \subset \mathfrak{f}_{2} \subset \ldots \ldots c \mathfrak{f}_{k-1} \subset \mathfrak{f}_{k}=\mathfrak{m} .
$$

In order to avoid trivialities we assume that all inclusions are strict. Thus, the length of the flag is the number of inclusions. A flag of length two simply is a proper subspace. A flag of length three will be called a short flag.

Two flags $\mathfrak{f}$ and $\mathfrak{f}^{\prime}$ of the same length $k$ are called transversal, and we write $\mathfrak{f} \top \mathfrak{f}^{\prime}$, if they are "crosswise complementary" in the following sense:

$$
\forall i=1, \ldots, k: \quad \mathfrak{f}_{i} \oplus \mathfrak{f}_{k-i}^{\prime}=\mathfrak{m} .
$$

If $\mathbb{K}$ is a field, then every flag admits at least one transversal flag. If $\mathbb{K}$ is just a ring, then this is not true in general. Therefore, we will only consider complemented flags, i.e. flags that admit a transversal flag. The set of all complemented flags of length $k$ will oftenly denoted by $\mathcal{F}=\mathcal{F}_{k}$. Obviously, $(\mathcal{F}, \mathcal{F} ; \top)$ is a pair geometry in the sense of Section 1.1. For $k=2$, by definition this geometry is called the Grassmann-geometry $(\mathcal{G} \operatorname{ras}(\mathfrak{m}), \mathcal{G} r a s(\mathfrak{m}) ; \top)$.

3.2. Gradings and filtrations. A $\mathbb{Z}$-grading of $\mathfrak{m}$ of length $k$ is a family $\mathfrak{g}=\left(\mathfrak{g}_{1}, \ldots, \mathfrak{g}_{k}\right)$ of submodules such that

$$
\mathfrak{m}=\mathfrak{g}_{1} \oplus \ldots \oplus \mathfrak{g}_{k} .
$$

Let us denote by $\mathcal{G}=\mathcal{G}_{k}$ the set of all gradings of $\mathfrak{m}$ of length $k$. Each grading $\mathfrak{g}=\left(\mathfrak{g}_{1}, \ldots, \mathfrak{g}_{k}\right)$ defines a pair of transversal flags of length $k$ : for $j=1, \ldots, k$ let

$$
\mathfrak{f}_{j}^{+}:=\mathfrak{g}_{1} \oplus \ldots \oplus \mathfrak{g}_{j}, \quad \mathfrak{f}_{k-j}^{-}:=\mathfrak{g}_{j+1} \oplus \ldots \oplus \mathfrak{g}_{k} .
$$

Obviously, $\mathfrak{f}_{j}^{+} \oplus \mathfrak{f}_{k-j}^{-}=\mathfrak{g}_{1} \oplus \ldots \oplus \mathfrak{g}_{k}=\mathfrak{m}$, whence the following flags are transversal

$$
\begin{aligned}
\mathfrak{f}^{+}:=\mathfrak{f}^{+}(\mathfrak{g}): & 0 \subset \mathfrak{f}_{1}^{+} \subset \mathfrak{f}_{2}^{+} \subset \ldots \subset \mathfrak{m}=\mathfrak{f}_{k}^{+} \\
\mathfrak{f}^{-}:=\mathfrak{f}^{-}(\mathfrak{g}): & 0 \subset \mathfrak{f}_{1}^{-} \subset \mathfrak{f}_{2}^{-} \subset \ldots \subset \mathfrak{m}=\mathfrak{f}_{k}^{-} .
\end{aligned}
$$

Observe that the grading $\mathfrak{g}$ can be recovered from $\left(\mathfrak{f}^{+}, \mathfrak{f}^{-}\right)$via $\mathfrak{g}_{j}=\mathfrak{f}_{j}^{+} \cap \mathfrak{f}_{k+1-j}^{-}($for $j=1, \ldots, k)$. 
Proposition 3.3. $\quad$ Two flags $\mathfrak{e}, \mathfrak{f}$ of length $k$ in a $\mathbb{K}$-module $\mathfrak{m}$ are transversal if and only if they are derived from a grading of $\mathfrak{m}$, i.e., if and only if there exists a grading $\mathfrak{g}$ such that $\mathfrak{e}=\mathfrak{f}^{+}(\mathfrak{g})$ and $\mathfrak{f}=\mathfrak{f}^{-}(\mathfrak{g})$. If $\mathfrak{e}$ and $\mathfrak{f}$ are transversal, then the corresponding grading of $\mathfrak{m}$ is given by $\mathfrak{g}_{j}=\mathfrak{e}_{j} \cap \mathfrak{f}_{k+1-j}$.

Proof. As already remarked, it is clear that flags derived from a grading are transversal. Let us prove the converse by induction on $k$. For $k=2$ the claim is trivial. We assume now that, for transversal flags of length $k$ in arbitrary $\mathbb{K}$-modules, it has already been shown that $\mathfrak{g}_{j}=\mathfrak{e}_{j} \cap \mathfrak{f}_{k+1-j}$ defines a grading. Given two transversal flags $\mathfrak{e}$ and $\mathfrak{f}$ of length $k+1$ in a $\mathbb{K}$-module $\mathfrak{m}$, let us take intersections with $\mathfrak{n}:=\mathfrak{e}_{k}$. Putting $\mathfrak{f}_{j}^{\prime}:=\mathfrak{f}_{j} \cap \mathfrak{e}_{k}$, we consider two flags of lenght $k$ in the space $\mathfrak{n}$, namely

$$
\begin{aligned}
& \mathfrak{e}_{1} \subset \mathfrak{e}_{2} \subset \ldots \subset \mathfrak{e}_{k-1} \subset \mathfrak{e}_{k}=\mathfrak{n}, \\
& 0=\mathfrak{f}_{1}^{\prime} \subset \mathfrak{f}_{2}^{\prime} \subset \ldots \subset \mathfrak{f}_{k}^{\prime} \subset \mathfrak{n},
\end{aligned}
$$

Let us show that these two flags are transversal, i.e., that

$$
\forall j=1, \ldots, k-1: \quad \mathfrak{e}_{k}=\mathfrak{e}_{j} \oplus\left(\mathfrak{f}_{k+1-j} \cap \mathfrak{e}_{k}\right) .
$$

First of all, the sum on the right hand side is direct since $\mathfrak{e}_{j} \cap \mathfrak{f}_{k+1-j}=0$. The inclusion " $\supset$ " is clear since $\mathfrak{e}_{j} \subset \mathfrak{e}_{k}$, and the inclusion " $\subset$ " follows since every element $x \in \mathfrak{m}$ can be decomposed as $x=x_{1}+x_{2}$ with $x_{1} \in \mathfrak{e}_{j}$ and $x_{2} \in \mathfrak{f}_{k+1-j}$, therefore, if $x \in \mathfrak{e}_{k}$, it follows that $x_{2}=x-x_{1}$ belongs both to $\mathfrak{f}_{k+1-j}$ and to $\mathfrak{e}_{k}$. Applying the induction hypothesis to these two transversal flags of length $k$, it follows that

$$
\mathfrak{e}_{k}=\bigoplus_{j=1}^{k}\left(\mathfrak{e}_{j} \cap \mathfrak{f}_{k+2-j} \cap \mathfrak{e}_{k}\right)=\bigoplus_{j=1}^{k}\left(\mathfrak{e}_{j} \cap \mathfrak{f}_{k+2-j}\right) .
$$

Finally, we have that $\mathfrak{m}=\mathfrak{f}_{1} \oplus \mathfrak{e}_{k}=\oplus_{j=1}^{k+1}\left(\mathfrak{e}_{j} \cap \mathfrak{f}_{k+2-j}\right)$.

3.4. Groups and matrix notation. Given a flag $\mathfrak{f}$ of length $k$, we define the stabilizer group $P(\mathfrak{f})$ ("parabolic") and its "unipotent radical" $U(\mathfrak{f})$ by

$$
\begin{aligned}
& P(\mathfrak{f})=\left\{g \in \mathrm{GL}(\mathfrak{m}) \mid \forall i: g\left(\mathfrak{f}_{i}\right)=\mathfrak{f}_{i}\right\}, \\
& U(\mathfrak{f})=\left\{g \in \mathrm{GL}(\mathfrak{m}) \mid \forall i:(g-\mathrm{id})\left(\mathfrak{f}_{i}\right) \subset \mathfrak{f}_{i-1}\right\} .
\end{aligned}
$$

For a given grading $\mathfrak{m}=\mathfrak{g}_{1} \oplus \ldots \oplus \mathfrak{g}_{k}$, we write endomorphisms $\alpha$ of $\mathfrak{m}$ as $k \times k$-matrices

$$
\left(A_{i j}\right)_{i, j=1, \ldots, k}, \quad A_{i j}=\operatorname{pr}_{i} \circ \alpha \circ \iota_{j}: \mathfrak{g}_{j} \rightarrow \mathfrak{g}_{i},
$$

with projections $\operatorname{pr}_{i}: \mathfrak{m} \rightarrow \mathfrak{g}_{i}$ and injections $\iota_{j}: \mathfrak{g}_{j} \rightarrow \mathfrak{m}$. Then, if $\mathfrak{f}$ is a fixed filtration of length $k$, and fixing an arbitrary transversal filtration $\mathfrak{e}$ (hence a grading, according to Prop. 3.3), elements of the group $P(\mathfrak{f})$ are represented by upper triangular matrices with invertible diagonal entries, and elements of $U(\mathfrak{f})$ are represented by upper triangular matrices with diagonal entries $\operatorname{id}_{\mathfrak{g}_{i}}, i=1, \ldots, k$. (Sometimes it will also be useful to consider the flag $\mathfrak{e}$ as "ascending" and $\mathfrak{f}$ as "descending"; then the groups $U(\mathfrak{f})$ and $P(\mathfrak{f})$ will be represented by lower triangular matrices.) We define also the "Lie algebra of $P(\mathfrak{f})$, resp. $U(\mathfrak{f})$ " by

$$
\begin{aligned}
& \mathfrak{p}(\mathfrak{f}):=\left\{X \in \operatorname{End}(\mathfrak{m}) \mid \forall i: X\left(\mathfrak{f}_{i}\right) \subset \mathfrak{f}_{i}\right\}, \\
& \mathfrak{u}(\mathfrak{f}):=\left\{X \in \operatorname{End}(\mathfrak{m}) \mid \forall i: X\left(\mathfrak{f}_{i}\right) \subset \mathfrak{f}_{i-1}\right\} .
\end{aligned}
$$

Linear operators $X \in \mathfrak{u}(\mathfrak{f})$ are nilpotent: $X^{k}=0$. Hence, if the integers $2, \ldots, k-1$ are invertible in $\mathbb{K}$, the exponential map

$$
\exp : \mathfrak{u}(\mathfrak{f}) \rightarrow U(\mathfrak{f}), \quad X \mapsto \exp (X)=\sum_{i=0}^{k-1} \frac{X^{i}}{i !}
$$

is a bijection whose inverse is given by $\log (\mathbf{1}+Y)=-\sum_{i=1}^{k-1} \frac{(-Y)^{i}}{i}$. 
Theorem 3.5 (The linear pair structure on flag geometries). Let $\mathcal{F}_{k}$ be the flag geometry of all complemented flags of length $k$ in a $\mathbb{K}$-module $\mathfrak{m}$.

(1) Given a flag $\mathfrak{f} \in \mathcal{F}_{k}$, the group $U(\mathfrak{f})$ acts simply transitively on the set $\mathfrak{f}^{\top}$ of flags that are transversal to $\mathfrak{f}$.

(2) Assume that $2,3, \ldots, k-1$ are invertible in $\mathbb{K}$. Then the flag geometry $\left(\mathcal{X}, \mathcal{X}^{\prime}\right)=\left(\mathcal{F}_{k}, \mathcal{F}_{k}\right)$ is a linear pair geometry over $\mathbb{K}$, where for a transversal pair $(\mathfrak{f}, \mathfrak{e})$ the linear structure on $\left(\mathfrak{f}^{\top}, \mathfrak{e}\right)$ is defined by declaring the bijection

$$
\mathfrak{u}(\mathfrak{f}) \rightarrow \mathfrak{f}^{\top}, \quad X \mapsto \exp (X) \cdot \mathfrak{e}
$$

to be a linear isomorphism.

(3) The linear pair geometry defined in part (2) is an affine pair geometry if $\left(\mathcal{X}, \mathcal{X}^{\prime}\right)$ is a Grassmann geometry, i.e. if $k=2$.

Proof. (1) We prove the claim by induction on the length $k$ of the flag: for $k=2$ (complements of a single subspace) the assertion is well-known: in this case $\mathfrak{f}^{\top}$ is an affine space on which the vector group $U(\mathfrak{f})$ acts as translation group. Next, suppose that the claim is proved for flags of length $k$. Consider a flag $\mathfrak{f}$ of length $k+1$ together with two flags $\mathfrak{e}$ and $\mathfrak{e}^{\prime}$ which are transversal to $\mathfrak{f}$. As both $\mathfrak{e}_{1}$ and $\mathfrak{e}_{1}^{\prime}$ are complements of $\mathfrak{f}_{k}$, we find $u_{1} \in \mathrm{GL}(\mathfrak{m})$ with $u_{1}\left(\mathfrak{e}_{1}\right)=\mathfrak{e}_{1}^{\prime}$ and $\left.u_{1}\right|_{\mathfrak{f}_{k}}=\mathrm{id}_{\mathfrak{f}_{k}}$. In particular, $u_{1}$ is an element of $U(\mathfrak{f})$. Now consider the flag $\tilde{\mathfrak{f}}$ of length $k$

$$
0 \subset \mathfrak{f}_{1} \subset \ldots \subset \mathfrak{f}_{k}=: \mathfrak{n} .
$$

Both flags $\tilde{\mathfrak{e}}_{j}:=\mathfrak{e}_{j} \cap \mathfrak{f}_{k}$ and $\widetilde{\mathfrak{e}}_{j}^{\prime}:=\mathfrak{e}_{j}^{\prime} \cap \mathfrak{f}_{k}(j=2, \ldots, k+1)$ are transversal to $\tilde{\mathfrak{f}}$, cp. see proof of Prop. 3.3. By the induction hypothesis, there exists an element $u_{2} \in U(\widetilde{f})$ with $u_{2}(\widetilde{\mathfrak{e}})=\widetilde{\mathfrak{e}}^{\prime}$. Therefore, $u_{3}:=\mathrm{id}_{\mathfrak{e}_{1}} \oplus u_{2}$ belongs to $U(\mathfrak{f})$ and satisfies $u_{3}\left(\widetilde{\mathfrak{e}}_{j}\right)=\widetilde{\mathfrak{e}}_{j}^{\prime}$. In matrix form, the elements $u_{1}$ and $u_{3}$ of $U(\mathfrak{f})$ are given by

$$
u_{1}=\left(\begin{array}{cc}
\operatorname{id}_{\mathfrak{e}_{1}} & * * \\
0 & \operatorname{id}_{\mathfrak{f}_{k}}
\end{array}\right), \quad u_{3}=\left(\begin{array}{cc}
\operatorname{id}_{\mathfrak{e}_{1}} & 0 \\
0 & u_{2}
\end{array}\right) .
$$

Since $\mathfrak{e}_{j}=\widetilde{\mathfrak{e}}_{j} \oplus \mathfrak{e}_{1}$ and $\mathfrak{e}_{j}^{\prime}=\widetilde{\mathfrak{e}}_{j}^{\prime} \oplus \mathfrak{e}_{1}$, we have $u_{3}\left(\widetilde{\mathfrak{e}}_{j}\right)=\widetilde{\mathfrak{e}}_{j}^{\prime}$ for $j=2, \ldots, k$. Finally, $u:=u_{1} \circ u_{3}$ is an element of $U(\mathfrak{f})$ such that $u(\mathfrak{e})=\mathfrak{e}^{\prime}$.

Uniqueness: Assume $u \in U(\mathfrak{f})$ is such that $u(\mathfrak{e})=\mathfrak{e}$. Then the matrix of $u$ clearly is the identity matrix, and hence $u=\mathrm{id}_{\mathfrak{m}}$.

(2) The exponential map $\mathfrak{u}(\mathfrak{f}) \rightarrow U(\mathfrak{f})$ being a bijection, composition with the bijection $U(\mathfrak{f}) \rightarrow \mathfrak{f}^{\top}, g \mapsto g \cdot \mathfrak{e}$ from part (1) yields a bijection between $\mathfrak{f}^{\top}$ and the $\mathbb{K}$-module $\mathfrak{u}(\mathfrak{f})$, which we use to define a linear structure on $\left(\mathfrak{f}^{\top}, \mathfrak{e}\right)$. Similarly, we define the linear structure on $\left(\mathfrak{e}^{\top}, \mathfrak{f}\right)$, and thus define a linear pair geometry.

(3) The linear pair geometry just defined is an affine pair geometry if the groups $U(\mathfrak{f})$ are all abelian, and this is the case if $k=2$.

3.6. Fixing the type. In general, the whole flag geometry $(\mathcal{F}, \mathcal{F})$ is neither connected (cf. 1.7) nor homegeoneous. Similar to Grasmmanian geometry, we therefore fix a type o (some flag of length $k$ ) and a co-type $\mathfrak{o}^{\prime}$ (some transversal flag), and then put

$$
\begin{aligned}
& \mathcal{X}:=\mathcal{F}_{\mathfrak{o}}^{\mathfrak{o}^{\prime}}:=\left\{\mathfrak{f} \in \mathcal{F} \mid \mathfrak{f} \cong \mathfrak{o}, \exists \mathfrak{f}^{\prime}: \mathfrak{f}^{\prime} \cong \mathfrak{o}^{\prime}, \mathfrak{f}^{\prime} \top \mathfrak{f}\right\}, \\
& \mathcal{X}^{\prime}:=\mathcal{F}_{\mathfrak{o}^{\prime}}^{\mathfrak{o}}:=\left\{\mathfrak{f} \in \mathcal{F} \mid \mathfrak{f} \cong \mathfrak{o}^{\prime}, \exists \mathfrak{f}^{\prime}: \mathfrak{f}^{\prime} \cong \mathfrak{o}, \mathfrak{f}^{\prime} \top \mathfrak{f}\right\},
\end{aligned}
$$

where $\mathfrak{f} \cong \mathfrak{e}$ means that $\mathfrak{f}_{j}$ and $\mathfrak{e}_{j}$ are isomorphic as $\mathbb{K}$-modules for all $j$. Note that, if $\mathfrak{f}$ is some flag, then all transversal flags $\mathfrak{f}^{\prime}$ of $\mathfrak{f}$ have necessarily the same type and co-type. Thus, if the 
intersection of $\mathfrak{f}^{\top}$ with $\mathcal{F}_{\mathfrak{o}^{\prime}}^{\mathfrak{o}}$ is non-empty, then necessarily the whole set $\mathfrak{f}^{\top}$ belongs to $\mathcal{F}_{\mathfrak{o}^{\prime}}^{\mathfrak{o}}$ and, consequently, $\left(\mathcal{X}, \mathcal{X}^{\prime}\right)=\left(\mathcal{F}_{\mathfrak{o}}^{\mathfrak{o}^{\prime}}, \mathcal{F}_{\mathfrak{o}^{\prime}}^{\mathfrak{o}}\right)$ forms a subgeometry of $(\mathcal{F}, \mathcal{F})$. If $\mathbb{K}$ is a field and $\mathfrak{m}=\mathbb{K}^{n}$, then $\mathcal{X}$ and $\mathcal{X}^{\prime}$ are spaces of flags of a given sequence of dimensions, and if moreover $k=2$ we get the usual Grassmannians of $p$-, resp. $q$-spaces in $\mathbb{K}^{n}$.

3.7. Remark: the structure of the structure maps. In the case of the Grassmann geometry one can give a fairly explicit formula for the structure maps $\boldsymbol{\Pi}_{r}$, and one can find algebraic identities that, in some sense, characterize these maps (see [Be02], [Be04]). For $k>2$, no results of this kind seem to be known. In this case, the linear parts $\alpha^{\top}$ carry many different affine structures (since the linear spaces $\left(\alpha^{\top}, x\right)$ for various choices of the origin $x$ in $\alpha^{\top}$ have no longer the same underlying affine space). A situation where one has to take account of many affine structures on the same underlying set occurs also in the theory of linear connections as developed in $[\mathrm{Be} 06]$ - we believe that these similarities are no fortuities. The collection of the various affine structures on $\alpha^{\top}$ contains more information than just the group structure on $\alpha^{\top}$, and one may expect that, as in the case $k=2$, this richer structure finally allows to develop the corresponding theory over rings and in infinite dimension, where the combinatorial or Lie theoretic information alone is too weak.

Theorem 3.8 (Standard intrinsic subspaces of flag geometries). Assume $\left(\mathcal{X}^{+}, \mathcal{X}^{-}\right)$is the flag geometry $(\mathcal{F}, \mathcal{F})$ of complemented flags of length $k$ in $\mathfrak{m}$ (or one of its subgeometries obtained by fixing type and co-type of the flags). We fix an index $j \in\{0, \ldots, k-1\}$ and some subspace $\mathfrak{e} \subset \mathfrak{m}$. Then the set

$$
\mathcal{I}_{\mathfrak{e} ; j}:=\left\{\mathfrak{f} \in \mathcal{X}^{+} \mid \mathfrak{f}_{j} \subset \mathfrak{e} \subset \mathfrak{f}_{j+1}\right\}
$$

is an intrinsic subspace of $\mathcal{X}^{+}$. Intrinsic subspaces of this type, as well as the intersections of finitely many of them, will be called standard intrinsic subspaces of the flag geometry.

Proof. Fixing terminology, we say that a flag $\mathfrak{f}$ is governed by the subspace $\mathfrak{e}$ in position $j$, written $\mathfrak{f}^{j} \mathfrak{e}$, if $\mathfrak{f}_{j} \subset \mathfrak{e} \subset \mathfrak{f}_{j+1}$. Let us prove that the set $\mathcal{I}:=\mathcal{I}_{\mathfrak{e}, j}=\left\{\mathfrak{f} \in \mathfrak{X} \mid \mathfrak{f}^{j} \prec \mathfrak{e}\right\}$ of flags governed by the subspace $\mathfrak{e}$ in position $j$ is an intrinsic subspace. If $\mathcal{I}$ is empty, there is nothing to prove. Thus, let $\mathfrak{o} \in \mathcal{I}$ and pick up some $\mathfrak{o}^{\prime} \in \mathcal{X}^{-}$with $\mathfrak{o} T \mathfrak{o}^{\prime}$. Then the theorem follows from the following

Claim 3.9. The set

$$
I:=\left\{u \in U\left(\mathfrak{o}^{\prime}\right) \mid u \cdot \mathfrak{o}^{j} \prec \mathfrak{e}\right\}
$$

is a subgroup of $U\left(\mathfrak{o}^{\prime}\right)$; more precisely, this group is the intersection of $U\left(\mathfrak{o}^{\prime}\right)$ with the "parabolic subgroup" $P(\mathfrak{e})$ stabilizing the subspace $\mathfrak{e}$.

Indeed, if Claim 3.9 is true, then the simple transitivity of the group $U\left(\mathfrak{o}^{\prime}\right)$ on $\mathfrak{v}_{\mathfrak{o}^{\prime}}$ shows that its subgroup $I$ acts simply transitively on $\mathcal{I} \cap \mathfrak{v}_{\mathfrak{o}^{\prime}}=I . \mathfrak{o}$. As the "Lie algebra" of $I$ (i.e., the image of $I$ under the logarithm $\left.\log : U\left(\mathfrak{o}^{\prime}\right) \rightarrow \mathfrak{u}\left(\mathfrak{o}^{\prime}\right)\right)$ is a linear space, given by $\mathfrak{u}\left(\mathfrak{o}^{\prime}\right) \cap \mathfrak{p}(\mathfrak{e})$, the set $I . \mathfrak{o}$ is a linear subspace of the linear space defined in Theorem 3.5 (2).

It remains to prove Claim 3.9. Notice that the relation $\mathfrak{o}^{j}<\mathfrak{e}$ is invariant under elements $g$ of the projective group of $\mathfrak{m}$, i.e. $\mathfrak{o}^{j} \prec \mathfrak{e}$ holds if and only if $g \cdot \mathfrak{o}^{j} \prec g \cdot \mathfrak{e}$. Therefore, $u \in P(\mathfrak{e})$ implies that $u . \mathfrak{o} \prec \mathfrak{e}$ and we derive that $P(\mathfrak{e}) \cap U\left(\mathfrak{o}^{\prime}\right) \subset I$. We shall show the converse inclusion $I \subset P(\mathfrak{e}) \cap U\left(\mathfrak{o}^{\prime}\right)$ by an explicit "matrix calculation": let $u$ be an element of $I$. We have to prove that $u$ is an element of $P(\mathfrak{e})$, i.e. that $u(\mathfrak{e}) \subset \mathfrak{e}$. As $u$ is an element of $U\left(\mathfrak{o}^{\prime}\right)$, we may represent $u$ by a lower triangular matrix ${ }^{1}\left(A_{i, j}\right)$ with respect to the grading $\mathfrak{m}=\mathfrak{g}_{1} \oplus \ldots \oplus \mathfrak{g}_{k}$

1 Thus, thinking in terms of matrices, we consider $\mathfrak{o}$ as an ascending flag, and $\mathfrak{o}^{\prime}$ as a descending flag; since $U\left(\mathfrak{o}^{\prime}\right)$ preserves the descending flag, it is represented by lower triangular matrices. The flag $0 \subset \mathfrak{e} \subset \mathfrak{m}$ should also be considered as ascending. 
corresponding to $\left(\mathfrak{o}, \mathfrak{o}^{\prime}\right)$. As in the proof of Prop. 3.3, we have that $\mathfrak{e}=\mathfrak{o}_{j} \oplus\left(\mathfrak{e} \cap \mathfrak{g}_{j+1}\right)$. Recall that the condition $u . \mathfrak{o} \prec \mathfrak{e}$ means that

a) $u \cdot \mathfrak{o}_{j}=(u \cdot \mathfrak{o})_{j} \subset \mathfrak{e}$, and

b) $\mathfrak{e} \subset(u \cdot \mathfrak{o})_{j+1}=u \cdot \mathfrak{o}_{j+1}$.

In terms of matrices, Condition a) can be rephrased as follows: we represent $u$ by a $3 \times 3$-matrix with respect to the decomposition $\mathfrak{m}=\left(\mathfrak{g}_{1} \oplus \ldots \oplus \mathfrak{g}_{j}\right) \oplus \mathfrak{g}_{j+1} \oplus\left(\mathfrak{g}_{j+2} \oplus \ldots \oplus \mathfrak{g}_{k}\right)$. From the assumption $\mathfrak{o}_{j} \subset \mathfrak{e} \subset \mathfrak{o}_{j+1}$ (i.e. $\left.\left(\mathfrak{g}_{1} \oplus \ldots \oplus \mathfrak{g}_{j}\right) \subset \mathfrak{e} \subset\left(\mathfrak{g}_{1} \oplus \ldots \oplus \mathfrak{g}_{j+1}\right)\right)$, we see that condition a) is fulfilled if and only if $u$ is represented by a matrix

$$
u=\left(\begin{array}{ccc}
1 & 0 & 0 \\
B & 1 & 0 \\
0 & * & 1
\end{array}\right), \quad B=\left(\begin{array}{ccc}
* & \ldots & * \\
0 & \ldots & 0
\end{array}\right),
$$

where the matrix $B$ just symbolically visualizes the condition $\operatorname{im}\left(A_{j+1, s}\right) \subset \mathfrak{e}, 1 \leq s<j$. We claim that, similarly, condition b) is equivalent to

$$
u=\left(\begin{array}{ccc}
\mathbf{1} & 0 & 0 \\
* & \mathbf{1} & 0 \\
0 & C & \mathbf{1}
\end{array}\right), \quad C=\left(\begin{array}{cc}
0 & * \\
& \vdots \\
0 & *
\end{array}\right)
$$

where the matrix $C$ stands for the condition $\mathfrak{g}_{j+1} \cap \mathfrak{e} \subset \operatorname{ker}\left(A_{s,(j+1)}\right)$ for all $s \leq j$. In fact, if $\mathfrak{m}$ is free over $\mathbb{K}$ of finite rank $n$, then the assertion follows from a) by transposing, i.e. by applying the preceeding case to the dual space (where now ascending and descending flags change their rôle). In the general case, we argue as follows ${ }^{2}:$ if $y \in\left(\mathfrak{g}_{j+1} \cap \mathfrak{e}\right)$, then we

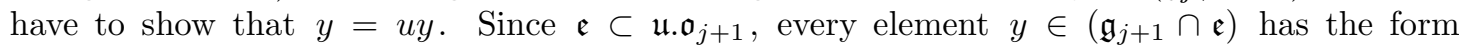
$y=u \cdot x=u \cdot \sum_{i \leq j+1} x_{i}=\sum_{i \leq j+1} x_{i}+\sum_{\substack{i \leq j+1 \\ s>i}} A_{i s} x_{i} \quad\left(\right.$ where $x_{i}=\operatorname{pr}_{i}(x) \in \mathfrak{g}_{i}$ ) for some $x \in \mathfrak{o}_{j+1}$. As $y \in \mathfrak{g}_{j+1}$ holds by assumption, this triangular system has the unique solution $x_{j+1}=y, x_{1}=0, \ldots, x_{j}=0$, whence $x=y$ and, hence, $y=u y$ as had to be shown.

Combining conditions a) and b), it is easy to see that $u$ satisfies the condition $u \cdot \mathfrak{e} \subset \mathfrak{e}$, and hence belongs to $U\left(\mathfrak{o}^{\prime}\right) \cap P(\mathfrak{e})$. This finishes the proof of the claim.

Typically, intersections of intrinsic subspaces of the type just described lead to the following situation: let $\mathfrak{e}$ be a flag of length $k+1$; then the set

$$
\mathcal{I}=\left\{\mathfrak{f} \in \mathcal{X} \mid \forall j=1, \ldots, k: \mathfrak{e}_{j} \subset \mathfrak{f}_{j} \subset \mathfrak{e}_{j+1}\right\}
$$

is an intrinsic subspace. Again, its linear image is the intersection of a group of unipotent lower triangular matrices with the "(upper triangular) parabolic subgroup" stabilizing the flag $\mathfrak{e}$.

3.10. Case of a Grassmann geometry. Let $\left(\mathcal{X}, \mathcal{X}^{\prime}\right)=\left(\mathcal{G r a s}_{\mathfrak{o}}^{\mathfrak{o}^{\prime}}(\mathfrak{m}), \mathcal{G} \operatorname{ras}_{\mathfrak{o}^{\prime}}^{\mathfrak{o}}(\mathfrak{m})\right)$ be a Grassmann geometry of fixed type and co-type. Fixing a flag of length three, $\mathfrak{e}=\left(0 \subset \mathfrak{e}_{1} \subset \mathfrak{e}_{2} \subset \mathfrak{m}\right)$, we consider the space

$$
\mathcal{I}_{\mathfrak{e}}:=\mathcal{I}_{\mathfrak{e}_{1} ; 0} \cap \mathcal{I}_{\mathfrak{e}_{2} ; 1}=\left\{x \in \mathcal{G} \operatorname{ras}(\mathfrak{m}) \mid \mathfrak{e}_{1} \subset x \subset \mathfrak{e}_{2}\right\} .
$$

With respect to a base point $\left(\mathfrak{o}, \mathfrak{o}^{\prime}\right)$, where $\mathfrak{o} \in \mathcal{I}_{\mathfrak{e}}$ (i.e., $\mathfrak{e}_{1} \subset \mathfrak{o} \subset \mathfrak{e}_{2}$ ), the space $I_{\mathfrak{e}}=\mathcal{I}_{e} \cap V$ is then given as follows: The group $U\left(\mathfrak{o}^{\prime}\right)$ is the abelian vector group

$$
U\left(\mathfrak{o}^{\prime}\right)=\left\{u=\left(\begin{array}{ll}
\mathbf{1} & 0 \\
f & \mathbf{1}
\end{array}\right) \mid f \in \operatorname{Hom}\left(\mathfrak{o}, \mathfrak{o}^{\prime}\right)\right\}
$$

\footnotetext{
2 One should expect that there is a general "duality principle" allowing to deal with such situations in an axiomatic context, such as the duality principle known in Jordan theory, see [Lo75, I Prop. 2.9]
} 
Then $u \cdot \mathfrak{o}=\Gamma_{f}$ can be identified with the graph $\Gamma_{f}=\left\{\left(\begin{array}{c}u \\ f u\end{array}\right) \mid u \in \mathfrak{o}\right\}$ of $f$, and (writing $\mathfrak{e}_{2}=\mathfrak{o} \oplus \mathfrak{e}_{2}^{\prime}$ with $\left.\mathfrak{e}_{2}^{\prime}=\mathfrak{e}_{2} \cap \mathfrak{o}^{\prime}\right)$ the compatibility conditions $\mathfrak{e}_{1} \subset \Gamma_{f}, \Gamma_{f} \subset \mathfrak{e}_{2}$, are equivalent to

$$
\mathfrak{e}_{1} \subset \operatorname{ker}(f), \quad \operatorname{im}(f) \subset \mathfrak{e}_{2}^{\prime} .
$$

In other words, $f \in I_{\mathfrak{e}_{1}, \mathfrak{e}_{2}^{\prime}}=\left\{f \in \operatorname{Hom}\left(\mathfrak{o}, \mathfrak{o}^{\prime}\right) \mid \mathfrak{e}_{1} \subset \operatorname{ker}(f), \operatorname{im}(f) \subset \mathfrak{e}_{2}^{\prime}\right\}$. Clearly, this is a linear subspace of $\operatorname{Hom}\left(\mathfrak{o}^{\prime}, \mathfrak{o}\right)$. Moreover, it is an inner ideal of $\operatorname{Hom}\left(\mathfrak{o}^{\prime}, \mathfrak{o}\right)$, see Appendix A, Section A.3. We will prove that, under suitable assumptions, all intrinsic subspaces are obtained in this way:

Theorem 3.11. Assume that $\mathbb{K}$ is an infinite field of characteristic not 2 and that $\left(\mathcal{X}^{+}, \mathcal{X}^{-}\right)$ is the Grassmannian geometry $\left(\mathcal{G r a s}_{p, q}(\mathbb{K}), \mathcal{G r a s}_{q, p}(\mathbb{K})\right)$ of $p$ - and $q$-spaces in a finite-dimensional vector space $\mathfrak{m} \cong \mathbb{K}^{p+q}$. Then all intrinsic subspaces of $\mathcal{X}^{+}$have the form $\mathcal{I}_{\mathfrak{e}}$ described in (3.2) for some flag of length three, $\mathfrak{e}=\left(\mathfrak{e}_{1} \subset \mathfrak{e}_{2} \subset \mathfrak{m}\right)$, such that $\operatorname{dim} \mathfrak{e}_{1} \leq p \leq \operatorname{dim} \mathfrak{e}_{2}$. The principal subspaces are precisely the intrinsic subspaces $\mathcal{I}_{\mathfrak{e}}$ with $p-\operatorname{dim} \mathfrak{e}_{1}=\operatorname{dim} \mathfrak{e}_{2}-p$.

Proof. Suppose that $\mathcal{I} \subset \mathcal{X}^{+}$is an intrinsic subspace; we may assume that it contains the base point $\mathfrak{o}=\mathbb{K}^{p}$. Let $\mathfrak{o}^{\prime} \top \mathfrak{o}$; we may assume that $\mathfrak{o}^{\prime}$ is the second component in the decomposition $\mathbb{K}^{n}=\mathbb{K}^{p} \oplus \mathbb{K}^{q}$. Let

$$
\left(V^{+}, V^{-}\right):=\left(\mathfrak{v}_{\mathfrak{o}^{\prime}}, \mathfrak{v}_{\mathfrak{o}}^{\prime}\right)=\left(\operatorname{Hom}\left(\mathfrak{o}, \mathfrak{o}^{\prime}\right), \operatorname{Hom}\left(\mathfrak{o}^{\prime}, \mathfrak{o}\right)\right)=(M(q, p ; \mathbb{K}), M(p, q ; \mathbb{K}))
$$

be the linearization of $\left(\mathcal{X}^{+}, \mathcal{X}^{-}\right)$corresponding to this base point. Then the affine part $I:=$ $\mathcal{I} \cap V^{+}$of $\mathcal{I}$ is a linear subspace of $V^{+}$.

We claim that $I$ is an inner ideal of $V^{+}=\operatorname{Hom}\left(\mathfrak{o}, \mathfrak{o}^{\prime}\right)$, i.e., $Q^{+}(I) V^{-} \subset I$, where $Q^{+}(X) Y=X Y X$ (cf. Appendix A, Section A.3). In order to prove this claim, let $X \in V^{+}$ and $Y \in V^{-}$such that $X$ and $-X$ lie in $\mathfrak{v}_{Y}$, i.e., $X \top Y$ and $-X \top Y$. (The set of such pairs $(X, Y)$ is non empty and Zariski-open in $V^{+} \times V^{-}$. This is seen by elementary arguments, or by using Jordan theory, noticing that that this condition is equivalent to saying that the pairs $(X, Y)$ and $(-X, Y)$ are quasi-invertible; in this case this means simply that $\mathbf{1}-X Y, \mathbf{1}-Y X$, $\mathbf{1}+X Y$ and $\mathbf{1}+Y X$ are invertible matrices.) By a direct (but somewhat tricky) calculation one can show that the geometric midpoint of $X$ and $-X$ in the affinization by $Y$ is given by the formula

$$
\Pi_{\frac{1}{2}}(X, Y,-X)=X Y X=Q^{+}(X) Y .
$$

(see [Be04, Eqn. (2.15)]). Since $\mathcal{I}$ is an intrinsic subspace, it follows that, whenever $X$ and $-X$ belong to $I=\mathcal{I} \cap V$ and $Y \in \mathcal{X}^{-}$is any affinization such that $X \top Y$ and $-X \top Y$, the geometric midpoint $\Pi_{\frac{1}{2}}(X, Y,-X)$ belongs again to $\mathcal{I}$. Therefore, the preceding calculation shows that, whenever $X \in I$, then, for some Zariski-dense set of elements $Y \in V^{-}$, the element $Q^{+}(X) Y$ again belongs to $I$. Since $\mathbb{K}$ is infinite, it follows that $Q^{+}(X) V^{-} \subset I$, i.e., $I$ is an inner ideal.

Now, from Proposition A.4, we know that all inner ideals are of the form $I_{E, F}$ with subspaces $E \subset \mathfrak{o}, F \subset \mathfrak{o}^{\prime}$. Comparing with the description of the affine picture of the intrinsic subspace $\mathcal{I}_{\mathfrak{e}}$ above, we see that, for the flag $\mathfrak{e}=(E \subset \mathfrak{o} \oplus F \subset \mathfrak{m})$, the intrinsic subspaces $\mathcal{I}_{e}$ and $\mathcal{I}$ have the same affine picture, i.e., the same intersection with $V^{+}$. We claim that $\mathcal{I}_{\mathfrak{e}}$ and $\mathcal{I}$ also have the same "points at infinity", i.e., that we have in fact equality $\mathcal{I}=\mathcal{I}_{\mathfrak{e}}$. In fact, the preceding arguments show that, with respect to any affinization, $\mathcal{I}$ agrees with some standard intrinsic subspace. Thus, covering $\mathcal{I}$ by a finite number $n$ of affine charts, the affine pictures coincide with those of spaces $\mathcal{I}_{\mathfrak{e}_{i}}$ defined by $n$ short flags $\mathfrak{e}^{1}, \ldots, \mathfrak{e}^{n}$. Since the intersection of two chart domains is Zariski-dense in each of the affine parts and the flag is uniquely determined by the affine picture on this intersection, it follows then that $\mathfrak{e}^{i}=\mathfrak{e}^{j}=\mathfrak{e}$ for all $i, j$, and hence $\mathcal{I}=\mathcal{I}_{\mathfrak{e}}$. 
In particular, the theorem says that, under suitable assumptions, the collection of all intrinsic subspaces in a Grassmann geometry forms again a linear pair geometry, namely a flag geometry (of short flags). We do not know whether an analogue of this theorem holds for $k>2$, the problem being that we do not have enough knowledge on the structure maps of flag geometries that are not "of commutative Jordan type" - cf. Section 3.7.

\section{Lagrangian flag geometries}

4.1. Lagrangian flags. Assume $\beta$ is a strongly non-degenerate quadratic or skew-symmetric form on $\mathfrak{m}$, i.e., the map $\mathfrak{m} \rightarrow \mathfrak{m}^{*}, v \mapsto \beta(v, \cdot)$ is not only injective, but also surjective. A Lagrangian flag (of length $k$ ) is a flag $\mathfrak{f}$ such that

$$
\forall j=1, \ldots, k: \quad \mathfrak{f}_{j}^{\perp}=\mathfrak{f}_{k-j}
$$

We say that a Lagrangian flag $\mathfrak{f}$ is complemented if there exists a transversal Lagrangian flag $\mathfrak{e}$, and we denote by $\mathcal{L}_{k}=\mathcal{L}_{k}(\mathfrak{m})$ the set of all complemented Lagrangian flags of length $k$ in $\mathfrak{m}$.

Let us first discuss the case $k=2$ which is particularly important. In this case, $\mathfrak{f}=(0 \subset$ $\left.\mathfrak{f}_{1} \subset \mathfrak{m}\right)$, and $\mathfrak{f}_{1}$ is a Lagrangian subspace, i.e., $\mathfrak{f}_{1}=\left(\mathfrak{f}_{1}\right)^{\perp}$, which admits a complementary Lagrangian subspace $\mathfrak{e}_{1}$. We call $\mathcal{L}_{2}$ also the Lagrangian Grassmannian (manifold of all Lagrangian subspaces). In matrix form, $\beta$ can then be represented on $\mathfrak{m}=\mathfrak{f}_{1} \oplus \mathfrak{e}_{1}$ by

$$
\left(\begin{array}{ll}
0 & \alpha \\
\gamma & 0
\end{array}\right)
$$

with pairings $\alpha: \mathfrak{e}_{1} \times \mathfrak{f}_{1} \rightarrow \mathbb{K}, \gamma: \mathfrak{f}_{1} \times \mathfrak{e}_{1} \rightarrow \mathbb{K}$. Then two cases have to be distinguished:

(sy) $\beta$ is symmetric, i.e. $\alpha=\gamma$ (under identification of $\mathfrak{e}_{1} \times \mathfrak{f}_{1}$ with $\mathfrak{f}_{1} \times \mathfrak{e}_{1}$ )

(sw) $\beta$ is skew-symmetric, i.e. $\alpha=-\gamma$.

If $\mathbb{K}$ is a field and $\mathfrak{m}=\mathbb{K}^{n}$, then necessarily $n$ is even, $n=2 m$, and for $\alpha$ we may choose the standard scalar product on $\mathbb{K}^{m}$. The case (sym) is then also called the artinian case and the case (skew) the symplectic case. For general Lagrangian flags of length $k \in \mathbb{N}$ we distinguish :

(1) $k$ is even. Then $\mathfrak{f}_{\frac{k}{2}}^{\perp}=\mathfrak{f}_{\frac{k}{2}}$ is again a Lagrangian subspace, and all $\mathfrak{f}_{j}$ with $j \leq \frac{k}{2}$ are isotropic subspaces. Lagrangian flags can be constructred by choosing a flag of $\frac{k}{2}$ isotropic subspaces, and completing this flag by adding the $\frac{k}{2}$ orthogonal complements. Note that, if $\mathbb{K}$ is a field, $\mathfrak{m}=\mathbb{K}^{n}=\mathbb{K}^{2 m}$, and (as in case $k=2$ ) $\beta$ will be artinian or symplectic.

(2) $k$ is odd. In this case it is not necessary that Lagrangian subspaces exist; therefore the form may be of general signature (e.g., for a Lorentzian form: a Lagrangian flag must be of the form $\mathbb{K} v \subset v^{\perp} \subset \mathfrak{m}$ for an isotropic vector $\left.v\right)$.

Theorem 4.2. The map

$$
\left(\mathcal{F}_{k}, \mathcal{F}_{k}\right) \rightarrow\left(\mathcal{F}_{k}, \mathcal{F}_{k}\right), \quad(\mathfrak{e}, \mathfrak{f}) \mapsto\left(\mathfrak{e}^{\perp}, \mathfrak{f}^{\perp}\right)
$$

is an automorphism of the flag geometry $\left(\mathcal{F}_{k}, \mathcal{F}_{k}\right)$ whose fixed point set is $\left(\mathcal{L}_{k}, \mathcal{L}_{k}\right)$. In particular, the Lagrangian geometry $\left(\mathcal{L}_{k}, \mathcal{L}_{k}\right)$ is a subspace of the linear pair geometry $\left(\mathcal{F}_{k}, \mathcal{F}_{k}\right)$. The linear pair geometry $\left(\mathcal{L}_{k}, \mathcal{L}_{k}\right)$ is an affine pair geometry if $k=2$.

Proof. By assumption, the map $b: \mathfrak{m} \rightarrow \mathfrak{m}^{*}, v \mapsto v^{*}=\beta(\cdot, v)$ is bijective. Since a decomposition $\mathfrak{m}=\mathfrak{a} \oplus \mathfrak{b}$ gives rise to a decomposition $\mathfrak{m}^{*}=\mathfrak{a}^{*} \oplus \mathfrak{b}^{*}$ (with $\mathfrak{b}^{*}$ corresponding 
to $\mathfrak{a}^{\perp}$ under the bijection $b$ ), it follows that $\mathfrak{e} \top \mathfrak{f}$ if and only if $\mathfrak{e}^{\perp} \top \mathfrak{f}^{\perp}$. Moreover, since $b$ is a bijection, the canonical map $\mathfrak{m} \rightarrow\left(\mathfrak{m}^{*}\right)^{*}$ into the double dual is also an isomorphism, and from this it follows that $\left(f^{\perp}\right)^{\perp}=\mathfrak{f}$.

Hence our map is an involution of the underlying pair geometry. It is an automorphism of linear pair geometries: for all $g \in \mathrm{GL}(\mathfrak{m})$ and all flags $\mathfrak{e}$, we have $(g \cdot \mathfrak{e})^{\perp}=\left(g^{*}\right)^{-1} \mathfrak{e}^{\perp}$ where $g^{*}$ is the adjoint of $g$ (the dual map of $g$ under the identification $b$ ). Thus the map

$$
V^{+}=\exp \left(\mathfrak{u}\left(\mathfrak{o}^{\prime}\right)\right) \cdot \mathfrak{o} \rightarrow\left(V^{+}\right)^{\perp}=\exp \left(\mathfrak{u}\left(\left(\mathfrak{o}^{\prime}\right)^{\perp}\right) \cdot \mathfrak{o}^{\perp}, \quad u \cdot \mathfrak{o} \mapsto\left(u^{*}\right)^{-1} \cdot \mathfrak{o}^{\perp}\right.
$$

is linear under the exponential map since it is given by $X \mapsto-X^{*}$.

Finally, since the fixed space of an automorphism is a subgeometry, it follows that $\left(\mathcal{L}_{k}, \mathcal{L}_{k}\right)$ is a subgeometry. For $k=2$ it is an affine pair geometry since it is a subspace of an affine pair geometry.

4.3. Symmetric matrices. Let us now focus on the case $k=2$. With respect to a fixed base point $\left(\mathfrak{o}, \mathfrak{o}^{\prime}\right)$ (i.e., a transversal pair of Lagrangian subspaces), the abelian group

$$
V^{+}=\mathrm{U}\left(\mathfrak{o}^{\prime}\right) \cap \mathrm{O}(\beta)=\left\{\left(\begin{array}{ll}
1 & v \\
0 & 1
\end{array}\right) \mid v \in \operatorname{Hom}(\mathfrak{o}, \mathfrak{o})\right\} \cap \mathrm{O}(\beta)
$$

is described as follows: using that $\beta$ defines a bijection $b: \mathfrak{m} \rightarrow \mathfrak{m}^{*}$, we get two bijections of $\mathfrak{o}$ with $\mathfrak{o}^{\prime}$, one of which is considered as an idenfication, i.e, we write $\mathfrak{m}=\mathfrak{o} \oplus \mathfrak{o}$. Then,

(sy) if $\beta$ is symmetric, both bijections coincide, i.e., we write $\beta=\left(\begin{array}{cc}0 & \alpha \\ \alpha & 0\end{array}\right)$; then $V^{+} \cong$ $\operatorname{Asym}(\mathfrak{o}, \alpha)$ is given by skewsymmetic operators $v$.

$(\mathrm{sw})$ if $\beta$ is skew-symmetric, we write $\beta=\left(\begin{array}{cc}0 & -\alpha \\ \alpha & 0\end{array}\right)$; then $V^{+} \cong \operatorname{Sym}(\mathfrak{o}, \alpha)$ is given by symmetric operators $v$.

In particular, symplectic Lagrangian geometries correspond to usual symmetric matrices, and artinian Lagrangian geometries correspond to usual skewsymmetric matrices. In the case $k>2$, similar (but more complicated) descriptions of the linear model space of the Lagrangian flags geometries can be given.

4.4. Standard intrinsic subspaces. Intersection of the standard intrinsic subspaces of the flag geometry $(\mathcal{F}, \mathcal{F})$ from Theorem 3.11 with the subgeometry $(\mathcal{L}, \mathcal{L})$ defines intrinsic subspaces in $\mathcal{L}$. For instance, for a fixed flag $\mathfrak{e}$ of length $k+1$, intersection of the intrinsic subspace defined by Equation (3.1) show that

$$
\mathcal{L}_{\mathfrak{e}}=\left\{\mathfrak{f} \in \mathcal{L} \mid \forall j=1, \ldots, k: \mathfrak{e}_{j} \subset \mathfrak{f}_{j} \subset \mathfrak{e}_{j+1}\right\}
$$

is an intrinsic subspace of $\mathcal{L}$. However, a condition $\mathfrak{e}_{j} \subset \mathfrak{f}_{j}$ automatically yields $\mathfrak{f}_{k-j}=\mathfrak{f}_{j}^{\perp} \subset \mathfrak{e}_{j}^{\perp}$, and therefore the intrinsic subspaces $\mathcal{L}_{\mathfrak{e}}$ are uninteresting unless $\mathfrak{e}$ itself was already a Lagrangian flag. For simplicity, assume now that $k=2$ and let $\mathfrak{e}=\left(0 \subset \mathfrak{e}_{1} \subset \mathfrak{e}_{1}^{\perp} \subset \mathfrak{m}\right)$ be a short Lagrangian flag. Then the affine image of the intrinsic subspace

$$
\mathcal{L}_{\mathfrak{e}}=\left\{\mathfrak{f}=\left(0 \subset \mathfrak{f}_{1} \subset \mathfrak{m}\right) \in \mathcal{L} \mid \mathfrak{e}_{1} \subset \mathfrak{f}_{1} \subset \mathfrak{e}_{1}^{\perp}\right\}
$$

is given as follows : write $\mathfrak{o}=\mathfrak{e}_{1} \oplus \mathfrak{a}$; then

$$
V_{\mathfrak{e}}=\{f \in \operatorname{Sym}(\mathfrak{o}) \mid \mathfrak{a} \subset \operatorname{ker} f\}=\left\{f \in \operatorname{Sym} \mid \operatorname{im} f \subset \mathfrak{a}^{\perp}\right\}
$$

(last equality: since $f$ is symmetric, we have $\operatorname{im}(f)=\operatorname{ker}(f)^{\perp}$, and hence the two conditions are equivalent). Then $V_{e}$ is an inner ideal in the Jordan algebra $\operatorname{Sym}(\mathfrak{o})$ (see Appendix A, A.12). If $\mathbb{K}$ is a field and $\mathfrak{o}=\mathbb{K}^{n}$, then one can show that every inner ideal is of this form (Appendix A.12), and we get the following analog of Theorem 3.11: 
Theorem 4.5. Assume that $\mathbb{K}$ is an infinite field of characteristic not 2 and that $\left(\mathcal{X}^{+}, \mathcal{X}^{-}\right)=$ $\left(\mathcal{L a g}_{2},{\mathcal{L} a g_{2}}\right)$ is a finite-dimensional symplectic Lagrangian geometry. Then all intrinsic subspaces of $\mathcal{X}^{+}$are of the form $\mathcal{I}_{\mathfrak{e}}$ given by (4.2), for some Lagrangian flag of length three, $\mathfrak{e}=\left(\mathfrak{e}_{1} \subset\right.$ $\left.\mathfrak{e}_{1}^{\perp} \subset \mathfrak{m}\right)$, such that $\operatorname{dim} \mathfrak{e}_{1} \leq n$. In this case, all intrinsic subspaces are principal.

Under the assumptions of the theorem, the geometry of intrinsic subspaces is essentially isomorphic to the geometry $\left(\mathcal{L}_{3}, \mathcal{L}_{3}\right)$ (subject to dimension conditions) and hence can be equipped with the structure of a linear pair geometry. Its connected compenents are homogeneous under the symplectic group $\operatorname{Sp}(n, \mathbb{K})$, with stabilizer groups being parabolic subgroups stabilizing some Lagrangian flag of length 3 . Finally, a similar resulat in the artinian case holds.

\section{Geometries of Lie algebra filtrations}

5.1. Filtered and graded algebras. Assume that $\mathfrak{m}$ carries the structure of an algebra over $\mathbb{K}$, with bilinear product denoted by $x \cdot y$. (It may be any product; only later we will assume that it is a Lie bracket and then we will write $[x, y]$.) Then we consider flags that are filtrations of this algebra, i.e., they are compatible with the product in the sense that $\mathfrak{f}_{i} \cdot \mathfrak{f}_{j} \subset f_{i+j}$. Similarly, we consider only gradings $\mathfrak{m}=\oplus_{i} \mathfrak{g}_{i}$ which are compatible with the algebra structure: $\mathfrak{g}_{i} \cdot \mathfrak{g}_{j} \subset \mathfrak{g}_{i+j}$. Note that, at this point, the choice of our indix set becomes important: so far we needed only that the index set is totally ordered; now we need a structure of abelian semigroup on the index set. Therefore we will no longer use $\{1, \ldots, k\}$ as index set, but rather speak of finite $\mathbb{Z}$-gradings $\mathfrak{m}=\oplus_{i \in \mathbb{Z}} \mathfrak{g}_{i}$, i.e., $\mathfrak{g}_{i} \neq 0$ only for finitely many indices, and similarly finite $\mathbb{Z}$-filtrations are defined.

Proposition 5.2. $\quad$ Let $\mathfrak{m}$ be an algebra over $\mathbb{K}$. Then the following objects are in canonical bijection with each other:

(1) pairs of transversal finite $\mathbb{Z}$-filtrations of $\mathfrak{m}$

(2) finite $\mathbb{Z}$-gradings of $\mathfrak{m}$

(3) derivations $D: \mathfrak{m} \rightarrow \mathfrak{m}$ which are diagonalizable over $\mathbb{Z}$ and have only finitely many eigenvalues which are contained in some intervall $-n, \ldots, n$ such that $1, \ldots, 3 n \in \mathbb{K}^{\times}$

Proof. It is clear that gradings give rise to pairs of transversal filtrations, and the converse follows from Prop. 3.3: with the notation introduced there, two transversal filtrations $\mathfrak{f}, \mathfrak{e}$ give rise, first of all to a $\mathbb{K}$-module grading of $\mathfrak{m}$ (Prop. 3.3), which is then easily seen to be an algebra grading.

Given a grading $\mathfrak{m}=\oplus_{i \in \mathbb{Z}} \mathfrak{g}_{i}$, one checks that the linear map $D$ given by $D(x)=i x$ for $x \in \mathfrak{g}_{i}$ is a derivation of the algebra $\mathfrak{m}$. Conversely, given a derivation as in (3), one checks that the eigenspace decomposition of $D$ is a finite $\mathbb{Z}$-grading. (The condition on the eigenvalues is to ensure that the eigenspace decomposition is direct.)

In general, the geometry of finite $\mathbb{Z}$-gradings of an algebra will not carry the structure of a linear pair geometry. However, under suitable assumptions this is the case. Let us assume that $\mathfrak{m}$ is a Lie algebra with bracket $[x, y]$, and let us consider 3 -filtrations: these are filtrations of length 3 , with index set $\{1,0,-1\}$, i.e., filtrations of the form

$$
\begin{aligned}
& 0 \subset \mathfrak{f}_{1} \subset \mathfrak{f}_{0} \subset \mathfrak{f}_{-1}=\mathfrak{m}, \\
& {\left[\mathfrak{f}_{1}, \mathfrak{f}_{1}\right]=0,\left[\mathfrak{f}_{0}, \mathfrak{f}_{0}\right] \subset \mathfrak{f}_{0},\left[\mathfrak{f}_{1}, \mathfrak{f}_{0}\right] \subset \mathfrak{f}_{1},\left[\mathfrak{m}, \mathfrak{f}_{1}\right] \subset \mathfrak{f}_{0},\left[\mathfrak{f}_{1}, \mathfrak{m}\right] \subset \mathfrak{f}_{0} .}
\end{aligned}
$$

Thus $\mathfrak{f}_{0}$ is a subalgebra, and $\mathfrak{f}_{1}$ is an abelian ideal in $\mathfrak{f}_{0}$; moreover, left multiplication $\operatorname{ad}(x)$ by elements $x \in \mathfrak{f}_{1}$ is three-step nilpotent. Thus, assuming that 2 and 3 are invertible in $\mathbb{K}$, there is a well-defined automorphism $e^{\text {ad(x)}}$ of $\mathfrak{g}$. In [BeNe04] the following is proved: 
Theorem 5.3. Assume that $\mathfrak{m}$ is a Lie algebra and 2 and 3 are invertible in $\mathbb{K}$. Then the geometry of all inner 3 -filtrations of $\mathfrak{g}$ carries a natural structure of an affine pair geometry over $\mathbb{K}$. More precisely, for every inner 3 -filtration $\mathfrak{f}=\left(\mathfrak{f}_{1} \subset \mathfrak{f}_{0} \subset \mathfrak{f}_{-1}=\mathfrak{m}\right)$, the vector group $\mathfrak{f}_{1}$ acts simply transitively on $\mathfrak{f}^{\top}$ via $(x, \mathfrak{e}) \mapsto e^{\operatorname{ad}(x)}(\mathfrak{e})$.

Here, the term "inner" means the following: a transversal pair $(\mathfrak{e}, \mathfrak{f})$ of 3 -filtrations is called inner, if the corresponding 3-grading $\mathfrak{m}=\mathfrak{g}_{1} \oplus \mathfrak{g}_{0} \oplus \mathfrak{g}_{-1}$ is of inner type, i.e. the derivation $D$ corresponding to this grading via Prop. 5.2 is an inner derivation: $D=\operatorname{ad}(E)$ for some element $E \in \mathfrak{m}$ (which is then called an Euler operator). Without this assumption the result does not hold: take, e.g., an abelian Lie algebra; then filtrations are just ordinary flags of length three; but the geometry of flags of length three is not an affine pair geometry. - We conjecture that also for general inner filtrations of the form $0 \subset \mathfrak{f}_{k} \subset \mathfrak{f}_{k-1} \subset \ldots \subset \mathfrak{f}_{-k}=\mathfrak{m}$ the (unipotent) group $\exp \left(\mathfrak{f}_{1}\right)$ acts simply transitively on $\mathfrak{f}^{\top}$, and that, therefore, such filtrations give rise to linear (non affine in general) pair geometries. This would be very interesting since a good deal of the theory from [BeNe04] and [BeNe05] would then also generalize, thus defining manifold structures on a class of flag geometries in arbitrary dimension and over general base fields and rings.

5.4. Fixing the type. As in Section 3.6, we restrict our attention to subgeometries of the geometry of all inner 3 -filtrations by fixing a type. In this case, this amounts to fix a base point $\left(\mathfrak{o}^{+}, \mathfrak{o}^{-}\right)$, which is nothing but a fixed pair of transversal inner 3 -filtrations, or, what is the same by Prop. 5.2, a fixed inner 3-grading $\mathfrak{m}=\mathfrak{g}_{1} \oplus \mathfrak{g}_{0} \oplus \mathfrak{g}_{-1}$, or a fixed Euler operator $E$. Then the subgroup $\operatorname{PE}(\mathfrak{m}, E)$ of $\operatorname{Aut}(\mathfrak{m})$ generated by $U^{+}=\exp \left(\mathfrak{g}_{1}\right)$ and by $U^{-}=\exp \left(\mathfrak{g}_{-1}\right)$ is called the elementary projective group of $(\mathfrak{m}, E)$. The pair of orbits

$$
\left(\mathcal{X}^{+}, \mathcal{X}^{-}\right), \quad \mathcal{X}^{ \pm}:=\operatorname{PE}(\mathfrak{m}, E) \cdot \mathfrak{o}^{ \pm}
$$

is then a subgeometry of the affine pair geometry from Theorem 5.3, called the projective geometry of the 3 -graded Lie algebra $(\mathfrak{m}, E)$.

5.5. Friendly Jordan pairs. If $\mathfrak{m}=\mathfrak{g}_{1} \oplus \mathfrak{g}_{0} \oplus \mathfrak{g}_{-1}$ is a 3 -graded Lie algebra (over a ring $\mathbb{K}$ in which 2 and 3 are invertible), then the pair $\left(V^{+}, V^{-}\right)=\left(\mathfrak{g}_{1}, \mathfrak{g}_{-1}\right)$ with the trilinear maps $T^{ \pm}(x, y, z):=-[[x, y], z]$ is called the associated Jordan pair (see Appendix B, Section B.2). It satisfies the identities of an abstract linear Jordan pair. Conversely, from a linear Jordan pair one can reconstruct a 3 -graded Lie algebra. We say that a Jordan par is friendly if it has the following property: for all $a \in V^{\mp}$, the sets

$$
U_{a}:=\left\{x \in V^{ \pm} \mid B^{ \pm}(x, a) \in \mathrm{GL}\left(V^{ \pm}\right)\right\}
$$

(where $B^{ \pm}(x, a) \in \operatorname{End}\left(V^{ \pm}\right)$is the Bergmann-Operator, see B.2) generate $V^{ \pm}$as a $\mathbb{K}$-module; an inner ideal $I \subset V^{+}$(see B.3) is called friendly if $\left(I, V^{-}\right)$is a friendly Jordan pair. For instance, finite dimensional Jordan pairs over infinite fields are friendly (because then $U_{a}$, being the complement of the zero set of the polynomial $\operatorname{det} B^{ \pm}(x, a)$, is Zariski-dense in $\left.V^{ \pm}\right)$; also, real and complex Banach Jordan pairs are friendly (since in these cases $U_{a}$ is an open neighborhood of the origin), and, more generally, all topological (C2)-Jordan pairs in the sense of [BeNe05, Section 5.1] are friendly (since also in this case $U_{a}$ is an open neighborhood of the origin). The sets $U_{a}$ essentially describe intersections of affine parts of $\mathcal{X}^{ \pm}$, and thus the friendlyness condition assures that such intersections are "not too small". This is important if one wants to construct some kind of manifold structure on $\mathcal{X}^{ \pm}$(see [BeNe05]).

Theorem 5.6 (Correspondence between subspaces and sub-Jordan pairs). Let $(\mathfrak{m}, E)$ be a 3 -graded Lie algebra, let $V^{ \pm}=\mathfrak{g}_{ \pm 1}$ be the associated Jordan pair and $\left(\mathcal{X}^{+}, \mathcal{X}^{-} ; \top\right)$ its associated projective geometry with base point $\left(\mathfrak{o}^{+}, \mathfrak{o}^{-}\right)$. We identify $V^{ \pm}$with the linear parts 
$\left(\mathfrak{o}^{\mp}\right)^{\top}=\exp \left(\mathfrak{g}_{ \pm 1}\right) \cdot \mathfrak{o}^{ \pm}$. Then we have the following correspondence between subspaces and subJordan pairs (resp., between intrinsic subspaces and inner ideals):

(1) If $\left(\mathcal{W}^{+}, \mathcal{W}^{-}\right)$is a subspace of the geometry $\left(\mathcal{X}^{+}, \mathcal{X}^{-}\right)$containing the base point $\left(o^{+}, o^{-}\right)$, then $\left(W^{+}, W^{-}\right):=\left(\mathcal{W}^{+} \cap V^{+}, \mathcal{W}^{-} \cap V^{-}\right)$is a Jordan sub-pair of the Jordan pair $\left(V^{+}, V^{-}\right)$, and if $\mathcal{I} \subset \mathcal{X}^{+}$is an intrinsic subspace containing the base point $o^{+}$, then $I:=\mathcal{I} \cap V^{+}$is an inner ideal of $V^{+}$.

(2) If $W=\left(W^{+}, W^{-}\right)$is a friendly sub-Jordan pair of $\left(V^{+}, V^{-}\right)$and $G_{W}$ the subgroup of $\mathrm{PE}(\mathfrak{g}, E)$ generated by $\exp \left(W^{+}\right)$and $\exp \left(W^{-}\right)$, then $\left(\mathcal{W}^{+}, \mathcal{W}^{-}\right):=\left(G_{W} \cdot \mathfrak{o}^{+}, G_{W} \cdot \mathfrak{o}^{-}\right)$is a subspace of $\left(\mathcal{X}^{+}, \mathcal{X}^{-}\right)$having $W$ as associated (via (1)) Jordan pair, i.e., $W^{ \pm}=\mathcal{W}^{ \pm} \cap V^{ \pm}$. If $I$ is a friendly inner ideal of $\left(V^{+}, V^{-}\right)$, then $\mathcal{I}:=G_{I} \cdot \mathfrak{o}^{+}$(where $G_{I}$ is the group generated by $\exp (I)$ and $\exp \left(V^{-}\right)$) is an intrinsic subspace such that $\mathcal{I} \cap V^{+}=I$.

Proof. (1) First of all, it follows from the very definition of a subspace (Section 2.1), that $\left(W^{+}, W^{-}\right)$is a pair of linear subspaces of $\left(V^{+}, V^{-}\right)$. It follows that $W^{ \pm}$can be naturally identified with its tangent space $T_{\mathfrak{o} \pm} W^{ \pm}$at the base point, where the tangent bundle $\left(T \mathcal{X}^{+}, T \mathcal{X}^{-}\right)$can be constructed either abstractly (as in [Be02], by using scalar extension of $\mathbb{K}$ by dual numbers) or directly in the flag model (as in [BeNe04, Section 2.1]). Now, the associated Jordan pair is constructed in a similar way as the Lie algebra of a subgroup of a Lie group (see [Be02]): the geometry $\left(\mathcal{X}^{+}, \mathcal{X}^{-}\right)$(and hence every subgeometry) is a generalized projective geometry $([\mathrm{Be} 02$, Th. 10.1]); for such a geometry, the associated Jordan pair is nothing but the pair of tangent spaces $\left(V^{+}, V^{-}\right)=\left(T_{o^{+}} \mathcal{X}^{+}, T_{o^{-}} \mathcal{X}^{-}\right)$, equipped with a multilinear product which is constructed by using the third order tangent bundle (essentially, this is an algebraic version of the construction of the associated Lie triple system of a symmetric space, see [Be06, Chapter 27]). If $\left(\mathcal{W}^{+}, \mathcal{W}^{-}\right)$is a subgeometry containg the base point, then the third order tangent bundle $\left(T^{3} \mathcal{W}^{+}, T^{3} \mathcal{W}^{-}\right)$is a subgeometry of $\left(T^{3} \mathcal{X}^{+}, T^{3} \mathcal{X}^{-}\right)$, and it follows that the pair of tangent spaces $\left(W^{+}, W^{-}\right) \cong\left(T_{o^{+}} \mathcal{W}^{+}, T_{o^{-}} \mathcal{W}^{-}\right)$is a sub-pair of the Jordan pair $\left(V^{+}, V^{-}\right)$. This proves the first statement, and the second statement is an immediate consequence since inner ideals are precisely the subpairs of the form $\left(I, V^{-}\right)$, and intrinsic subspaces are precisely the subspaces of the form $\left(\mathcal{W}^{+}, \mathcal{W}^{-}\right)$with $\mathcal{W}^{-}$containing the whole affine part $V^{-}$.

(2) Assume now that a friendly sub-pair $\left(W^{+}, W^{-}\right)$of $\left(V^{+}, V^{-}\right)$is given, and define $\mathcal{W}^{ \pm}=G_{W} \cdot \mathfrak{o}^{ \pm}$as in the claim. The main point of the proof of Part (2) is to show that $W^{ \pm}=\mathcal{W}^{ \pm} \cap V^{ \pm}$. (Note that this claim means that phenomena such as the "dense wind" known from Lie theory do not occur in our situation.) Now, this claim is a consequence of results from [BeNe04] and [BeNe05]: in [BeNe04, Th. 2.8] it is shown that the group $\mathrm{PE}(\mathfrak{g}, E)$ and hence also $G_{W}$ act by "Jordan fractional quadratic maps". This means that, whenever a point $p=g . o^{+} \in \mathcal{W}^{+}$belongs to the affine part $V^{+}$, we can write $g . o^{+}=d_{g}\left(o^{+}\right)^{-1} n_{g}\left(o^{+}\right)$with a polynomial $d_{g}: V^{+} \rightarrow \operatorname{End}\left(V^{+}\right)$called "denominator" and a polynomial $n_{g}: V^{+} \rightarrow V^{+}$ called "nominator". We claim that, if $g \in G_{W}$, the denominator $d_{g}\left(o^{+}\right)$always belongs to the associative subalgebra $\mathcal{B}$ of $\operatorname{End}\left(V^{+}\right)$generated by the Bergmann-Operators $B(x, a)$ with $x \in W^{+}, a \in W^{-}$. In fact, this is proved exactly as in [BeNe05, Lemma 5.4], by decomposing $g \in G_{W}$ into a finite product of elements of the form $\exp \left(x_{i}\right) \exp \left(y_{i}\right)$ with $x_{i} \in W^{+}$, $y_{i} \in W^{-}$, and by applying induction on the "length" of such a decomposition. It is precisely at this point that the friendlyness condition is needed (cf. [BeNe05, loc. cit.], where instead of friendlyness the stronger assumption of a topological $\mathrm{C} 2$-condition was made). Having shown that $d_{g}\left(\mathfrak{o}^{+}\right)$belongs to the algebra $\mathcal{B}$ defined above, it follows that $d_{g}\left(\mathfrak{o}^{+}\right)$preserves $W^{+}$ since Bergmann operators $B(x, a)$ with $x \in W^{+}, a \in W^{-}$preserve $W^{+}$because $\left(W^{+}, W^{-}\right)$ is sub-pair. The same arguments apply as well to the nominators: if $g \in G_{W}$, one proves by induction on the decomposition length of $g$ that $n_{g}\left(\mathfrak{o}^{+}\right)$belongs to $W^{+}$. Finally, we get that $g . o^{+}=d_{g}\left(o^{+}\right)^{-1} n_{g}\left(o^{+}\right)$also belongs to $W^{+}$, i.e. $\mathcal{W}^{+} \cap V^{+} \subset W^{+}$. The other inclusion being clear since $G_{W}$ contains translations by $W^{+}$, we have equality. Similarly, we see that $\mathcal{W}^{-} \cap V^{-}=W^{-}$. 
It follows now easily that $\left(\mathcal{W}^{+}, \mathcal{W}^{-}\right)$is indeed a subgeometry of $\left(\mathcal{X}^{+}, \mathcal{X}^{-}\right)$. In fact, since, by definition, $\mathcal{W}^{+}$and $\mathcal{W}^{-}$are homogeneous under $G_{W}$, for every transversal pair $(x, a) \in \mathcal{W}^{+} \times \mathcal{W}^{-}$, there exists $g \in G_{W}$ such that $g \cdot(x, a)=\left(\mathfrak{o}^{+}, \mathfrak{o}^{-}\right)$(cf. [Be02, Theorem 5.7]). Thus $\mathcal{W}^{+} \cap \mathfrak{v}_{a}$ is a linear subspace of $\left(\mathfrak{v}_{a}, x\right)$, since the same is true with respect to $\mathfrak{o}^{-}$, as we have just seen. Similarly, $\mathcal{W}^{-} \cap \mathfrak{v}_{x}^{\prime}$ is a linear subspace of $\left(\mathfrak{v}_{x}^{\prime}, a\right)$, and the claim on subspaces is proved. As above, the claim on intrinsic subspaces is an immediate consequence.

5.7. Geometries of intrinsic subspaces. In a sense, the preceding theorem shows that the "geometry of all intrinsic subspaces of $\mathcal{X}^{+}$" is the global object corresponding to the "geometry of inner ideals of $V^{+}$" (see Section 5.11 for further comments concerning the latter). The geometry of all intrinsic subspaces being too big, we will again "fix a type", i.e., we consider the orbit $G \cdot \mathcal{I}$ of a given intrinsic subspace $\mathcal{I}$ under the projective group $G=\operatorname{PE}(\mathfrak{g}, E)$ and consider the orbit as a homogeneous space $G \cdot \mathcal{I} \cong G / G_{\mathcal{I}}$, where $G_{\mathcal{I}}=\{g \in G \mid g(\mathcal{I})=\mathcal{I}\}$. If $\mathcal{I}$ corresponds to the inner ideal $I \subset V^{+}$, then (by construction in Theorem 5.6) $G_{\mathcal{I}}$ contains the group $G_{I}=\left\langle\exp (I), \exp \left(V^{-}\right)\right\rangle$. (It may be strictly bigger: take, e.g., $I=0$; then $G_{I}=\exp V^{-}$, whereas $G_{\mathcal{I}}$ is the semidirect product of $\exp V^{-}$with the structure group $\operatorname{Str}\left(V^{+}, V^{-}\right)$, and hence $G$.I is the point space $\mathcal{X}^{+}$. In the general case, $G_{\mathcal{I}}$ is generated by its subgroups $G_{I}$ and the linear group $S_{I}=:\left\{g \in \operatorname{Str}\left(V^{+}, V^{-}\right) \mid g^{+}(I)=I\right\}$.) As a first step, one may analyse these groups by determining their "Lie algebras"

$$
\begin{aligned}
\mathfrak{g}_{I} & =\left\langle I, \mathfrak{g}_{-1}\right\rangle=I \oplus\left[I, \mathfrak{g}_{-1}\right] \oplus \mathfrak{g}_{-1}, \\
\mathfrak{g}_{\mathcal{I}} & =I \oplus \mathfrak{s}_{I} \oplus \mathfrak{g}_{-1}, \quad \mathfrak{s}_{I}:=\left\{X \in \mathfrak{g}_{0} \mid[X, I] \subset I\right\}
\end{aligned}
$$

The type of intrinsic subspaces we are interested in is defined by the Peirce-decomposition associated to an idempotent, cf. Appendix A.7 and B.4.

Theorem 5.8 (Peirce ideals and 5-graded geometries). Assume $\left(V^{+}, V^{-}\right)$is a Jordan pair with corresponding 3 -graded Lie algebra $\mathfrak{m}=\mathfrak{g}_{1} \oplus \mathfrak{g}_{0} \oplus \mathfrak{g}_{-1}$ and $\left(e^{+}, e^{-}\right)$is a non-zero idempotent in $\left(V^{+}, V^{-}\right)$, with associated Peirce-decomposition $V^{+}=V_{2} \oplus V_{1} \oplus V_{0}$. We assume the inner ideal $I:=V_{2}$ to be friendly, and let $\mathcal{I}$ be the corresponding intrinsic subspace in $\mathcal{X}^{+}$.

(1) There exists a 5 -grading $\mathfrak{m}=\mathfrak{e}_{2} \oplus \mathfrak{e}_{1} \oplus \mathfrak{e}_{0} \oplus \mathfrak{e}_{-1} \oplus \mathfrak{e}_{-2}$ of $\mathfrak{m}$ such that the stabilizer algebra $\mathfrak{g}_{\mathcal{I}}$ is equal to the "parabolic" subalgebra $\mathfrak{q}$ associated to the minus-filtration of this 5 -grading, i.e.

$$
\mathfrak{g}_{\mathcal{I}}=\mathfrak{q}:=\mathfrak{e}_{0} \oplus \mathfrak{e}_{-1} \oplus \mathfrak{e}_{-2}
$$

(2) The group $G_{\mathcal{I}}$ is equal to the normalizer $N_{G}(\mathfrak{q})$ of $\mathfrak{q}$ in $G$. In other words, the homogeneous space G.I is isomorphic to the orbit G.q and also to the G-orbit of the minus-filtration of $\mathfrak{e}$.

Proof. Before constructing the 5 -grading (Lemma 5.9 below), let us introduce some terminology: we say that an inner 5 -grading $\mathfrak{m}=\mathfrak{h}_{2} \oplus \mathfrak{h}_{1} \oplus \mathfrak{h}_{0} \oplus \mathfrak{h}_{-1} \oplus \mathfrak{h}_{-2}$ of a Lie algebra $\mathfrak{m}$ is of Peirce type with respect to an inner 3 -grading $\mathfrak{m}=\mathfrak{g}_{1} \oplus \mathfrak{g}_{0} \oplus \mathfrak{g}_{1}$ if there exist $e^{+} \in \mathfrak{g}_{1}, e^{-} \in \mathfrak{g}_{-1}$, $H \in \mathfrak{g}_{0}$ such that $\operatorname{ad}(H)$ is the grading derivation of the 5 -grading and $e^{+}, H, e^{-}$is an $\mathfrak{s l}_{2}$-triple:

$$
\left[e^{+}, e^{-}\right]=H, \quad\left[H, e^{+}\right]=2 e^{+}, \quad\left[H, e^{-}\right]=-2 e^{-} .
$$

Lemma 5.9. Assume $\mathfrak{g}=\mathfrak{g}_{1} \oplus \mathfrak{g}_{0} \oplus \mathfrak{g}_{-1}$ is a 3 -graded Lie algebra with Euler operator $E$ associated to the Jordan pair $\left(V^{+}, V^{-}\right)=\left(\mathfrak{g}_{1}, \mathfrak{g}_{-1}\right)$.

(1) Every non-zero idempotent $\left(e^{-}, e^{+}\right)$gives rise to a 5 -grading that is of Peirce type (with respect to the given 3-grading), having $H=\left[e^{+}, e^{-}\right]$as grading element, and every 5 grading of Peirce type is obtained in this way. 
(2) If $E$ and $H$ are grading elements as above, then $\operatorname{ad}(E)$ and $\operatorname{ad}(H)$ are simultaneously diagonalisable, and only the following combinations of eigenvalues can appear:

$\begin{array}{lllccccccc}\operatorname{ad}(E) & -1 & -1 & -1 & 0 & 0 & 0 & 1 & 1 & 1 \\ \operatorname{ad}(H) & -2 & -1 & 0 & -1 & 0 & 1 & 0 & 1 & 2\end{array}$

(3) If $E$ and $H$ are grading elements as in (1), then the element $H^{\prime}:=2 E-H$ is again a grading element of a 5-grading $\mathfrak{g}=\mathfrak{e}_{-2} \oplus \ldots \oplus \mathfrak{e}_{2}$. In terms of the 5 -grading $\mathfrak{g}=$ $\mathfrak{h}_{2} \oplus \mathfrak{h}_{1} \oplus \mathfrak{h}_{0} \oplus \mathfrak{h}_{-1} \oplus \mathfrak{h}_{-2}$ from Part (1) and the 3 -grading $\mathfrak{g}=\mathfrak{g}_{1} \oplus \mathfrak{g}_{0} \oplus \mathfrak{g}_{-1}$, we have

$$
\begin{aligned}
\mathfrak{e}_{2} & =\mathfrak{h}_{0} \cap \mathfrak{g}_{1} & \mathfrak{e}_{1} & =\left(\mathfrak{h}_{1} \cap \mathfrak{g}_{1}\right) \oplus\left(\mathfrak{g}_{0} \cap \mathfrak{h}_{-1}\right) \\
\mathfrak{e}_{-2} & =\mathfrak{h}_{0} \cap \mathfrak{g}_{-1} & \mathfrak{e}_{-1} & =\left(\mathfrak{h}_{-1} \cap \mathfrak{g}_{-1}\right) \oplus\left(\mathfrak{g}_{0} \cap \mathfrak{h}_{1}\right)
\end{aligned}
$$

In particular, if $\mathfrak{o}=\mathfrak{o}(E)$, resp. $\mathfrak{f}=\mathfrak{f}\left(H^{\prime}\right)$, are the + -filtrations corresponding to the 3 (resp. 5)-grading just described, we have the relations

$$
\mathfrak{f}_{2} \subset \mathfrak{o}_{1} \subset \mathfrak{f}_{0}, \quad \mathfrak{f}_{1} \subset \mathfrak{o}_{0} \subset \mathfrak{f}_{-1} .
$$

(4) Assume that, moreover, the Jordan pair $\left(V^{+}, V^{-}\right)=(V, V)$ is associated to a Jordan algebra $V$ with unit 1 and that $\left(e^{+}, e^{-}\right)=(e, e)$ for an idempotent $e$ of $V$. Then the grading element $H^{\prime}$ defined in Part (3) comes again from an idempotent, namely from $(1-e, 1-e)$.

Proof. (1) Assume $\left(e^{+}, e^{-}\right)$is a non-zero idempotent in $\left(V^{+}, V^{-}\right)$and let $V^{+}=V_{0} \oplus V_{1} \oplus V_{2}$ be the associated Peirce decomposition. Let $H:=\left[e^{+}, e^{-}\right] \in \mathfrak{g}_{0}$; then we have $\left[H, e^{+}\right]=$ $\left[\left[e^{+}, e^{-}\right], e^{+}\right]=T\left(e^{+}, e^{-}, e^{+}\right)=2 Q\left(e^{+}\right) e^{-}=2 e^{+}$and $\left[H, e^{-}\right]=-2 e^{-}$, i.e., $e^{+}, H, e^{-}$is an $\mathfrak{s l}_{2}$-triple. The element $H$ acts diagonally on $V^{+}$, with possible eigenvalues $0,1,2$ and the eigenspaces given by the Peirce decomposition (cf. Appendix B, B.4 (1)), and since $H=$ $-\left[e^{-}, e^{+}\right]$, the same argument applied to $V^{-}$shows that $H$ acts diagonally on $V^{-}$with possible eigenvalues $0,-1,-2$. Since we assume that $\mathfrak{g}$ is generated by $\mathfrak{g}_{ \pm}=V^{ \pm}$and the Euler operator $E$, it follows that $H$ acts diagonally on $\mathfrak{g}_{0}=\sum_{i, j=-2}^{2}\left[\left(V^{+}\right)_{i},\left(V^{-}\right)_{j}\right]+\mathbb{K} E$, with possible eigenvalues $-4, \ldots, 4$. But since $V^{+}$and $V^{-}$are abelian, the brackets $\left[\left(V^{+}\right)_{i},\left(V^{+}\right)_{j}\right]$ etc. are zero, and the possible eigenvalues are among the integers $-2, \ldots, 2$. Summing up, the derivation $\operatorname{ad}(H): \mathfrak{g} \rightarrow \mathfrak{g}$ is diagonalizable with integer eigenvalues and defines a 5-grading of $\mathfrak{m}$ (cf. Prop. $5.2)$.

Conversely, given a 5 -grading of Peirce type, it follows from the relation $\left[\left[e^{ \pm}, e^{\mp}\right], e^{ \pm}\right]=2 e^{ \pm}$ that $\left(e^{+}, e^{-}\right)$is an idempotent, whose grading element is, by definition, $\left[e^{+}, e^{-}\right]$.

(2) Since $H=T\left(e^{+}, e^{-}\right)$belongs to $\mathfrak{g}_{0}, \operatorname{ad}(E)$ and $\operatorname{ad}(H)$ commute and hence are simultaneously diagonalizable. In view of the Peirce decomposition of $V^{+}=\mathfrak{g}_{1}$ and of $V^{-}=\mathfrak{g}_{-1}$ (where only the given three eigenvalues of $H$ appear), it remains only to prove that the eigenvalues 2 and -2 of $\operatorname{ad}(H)$ do not appear in $\mathfrak{g}_{0}$. The proof is merely a question of representation theory of the Lie algebra $\mathfrak{s l}_{2}$ : decomposing $\mathfrak{g}$ in isotopycal components under the $\mathfrak{s l}_{2}$-action, from the given eigenvalues one sees that only the irreducible representations of $\mathfrak{s l}_{2}$ on $\rho_{j}$ on $\mathbb{K}^{j+1}$ for $j=0,1,2$ can appear, where $\rho_{0}$ is the trivial representation, $\rho_{1}$ the natural representation by $2 \times 2$-matrices

$$
\rho_{1}(H)=\left(\begin{array}{cc}
1 & \\
& -1
\end{array}\right), \quad \rho_{1}\left(e^{+}\right)=\left(\begin{array}{ll}
0 & 1 \\
& 0
\end{array}\right), \quad \rho_{1}\left(e^{-}\right)=\left(\begin{array}{ll}
0 & \\
1 & 0
\end{array}\right),
$$

and $\rho_{2}$ the adjoint representation given by

$$
\rho_{2}(H)=\left(\begin{array}{ccc}
2 & & \\
& 0 & \\
& & -2
\end{array}\right), \quad \rho_{2}\left(e^{+}\right)=\left(\begin{array}{ccc}
0 & 2 & \\
& 0 & 1 \\
& & 0
\end{array}\right), \quad \rho_{2}\left(e^{-}\right)=\left(\begin{array}{ccc}
0 & & \\
1 & 0 & \\
& 2 & 0
\end{array}\right) .
$$


Now let $\mathfrak{k} \subset \mathfrak{g}$ be an irreducible $\mathfrak{s l}_{2}$-submodule of $\mathfrak{g}$ of type $\rho_{2}$. Let $v$ be a highest weight vector: $\left[e^{+}, v\right]=0,[H, v]=2 v$, and $v,\left[e^{-}, v\right],\left[e^{-},\left[e^{-}, v\right]\right]$ spans $\mathfrak{k}$ as a $\mathbb{K}$-module. Thus the lowest weight vector $w=\left[e^{-},\left[e^{-}, v\right]\right]$ belongs to $V^{-}=\mathfrak{g}_{-1}$, and the same argument shows that the highest weight vector $v$ belongs to $V^{+}=\mathfrak{g}_{1}$. Summing up, the highest weight vector always belongs to $V_{2}$, and hence eigenvectors of $\operatorname{ad}(H)$ for the eigenvalue 2 always belong to $\mathfrak{g}_{1}$ and never to $\mathfrak{g}_{0}$. In the same way, it is seen that eigenvectors of $\operatorname{ad}(H)$ for the eigenvalue -2 always belong to $\mathfrak{g}_{-1}$ and never to $\mathfrak{g}_{0}$. Since $\mathfrak{g}_{0}$ is $\operatorname{ad}(H)$-stable, we can write $\mathfrak{g}_{0}=\oplus_{i=-2}^{2}\left(\mathfrak{g}_{0} \cap \mathfrak{h}_{i}\right)$; the preceding arguments show that the first and last term are zero, and hence our claim follows.

(3) Clearly, $\operatorname{ad}(2 E-H)$ is again a derivation of $\mathfrak{g}$, and using Part (2), the eigenvalues are given by the following table:

$\begin{array}{cccccccccc}\operatorname{ad}(E) & -1 & -1 & -1 & 0 & 0 & 0 & 1 & 1 & 1 \\ \operatorname{ad}(H) & -2 & -1 & 0 & -1 & 0 & 1 & 0 & 1 & 2 \\ \operatorname{ad}(2 E-H) & 0 & -1 & -2 & 1 & 0 & -1 & 2 & 1 & 0\end{array}$

Thus $\operatorname{ad}(2 E-H)$ has again possible eigenvalues $-2,-1,0,1,2$, and moreover the table gives the eigenspace decomposition stated in the lemma. (We call $H^{\prime}$ the conjugate grading of $H$. Note that, passing to the conjugate grading, the eigenspaces belonging to 0 and \pm 2 in $\mathfrak{g}_{ \pm}$are exchanged, whereas those of \pm 1 remain unchanged.) The last relations are also an immediate consequence of eigenvalue combinations from the table (e.g., we have $\mathfrak{f}_{2}=\mathfrak{e}_{2}=V_{0} \subset V^{+}=\mathfrak{g}_{1}=$ $\mathfrak{o}_{1}$, and so on).

(4) If $e$ is an idempotent in a unital Jordan algebra, then $1-e$ is again an idempotent, having same Peirce 1-space, whereas the Peirce 0 -space and the Peirce 2 -space are exchanged (cf. [Lo75, I.5.6].). As explained above, this is exactly what happens in Part (3) for the conjugate grading, and hence we can conclude that $H^{\prime}=\operatorname{ad} T(1-e, 1-e)$.

Now we prove Part (1) of the theorem. We define the 5-grading $\mathfrak{g}=\mathfrak{e}_{2} \oplus \ldots \oplus \mathfrak{e}_{-2}$ as in part (3) of the lemma. Recall that the Lie algebra $\mathfrak{g}_{I}$ is generated by $V_{2}=\mathfrak{g}_{1} \cap \mathfrak{e}_{0}$ and $V^{-}=\mathfrak{g}_{-1}=\mathfrak{g}_{-1} \cap\left(\mathfrak{e}_{0} \oplus \mathfrak{e}_{-1} \oplus \mathfrak{e}_{-2}\right)$, i.e.,

$$
\mathfrak{g}_{I}=\left\langle\left(\mathfrak{g}_{1} \oplus \mathfrak{g}_{-1}\right) \cap\left(\mathfrak{e}_{0} \oplus \mathfrak{e}_{-1} \oplus \mathfrak{e}_{-2}\right)\right\rangle .
$$

Recall further that $\mathfrak{s}_{I}=\left\{X \in \mathfrak{g}_{0} \mid[X, I] \subset I\right\}$ is the normalizer of $I$ in $\mathfrak{g}_{0}$. We claim that

$$
\mathfrak{s}_{I}=\mathfrak{g}_{0} \cap\left(\mathfrak{e}_{0} \oplus \mathfrak{e}_{-1}\right) .
$$

The statement (1) follows then from (5.2) and (5.3) since $\mathfrak{g}_{\mathcal{I}}=\mathfrak{g}_{I}+\mathfrak{s}_{I}$. Let us prove (5.3): the inclusion " $\supset$ " follows directly from the commutation rules $\left[\mathfrak{e}_{i}, \mathfrak{e}_{j}\right] \subset \mathfrak{e}_{i+j}$ since $I=V_{2}=\mathfrak{e}_{0} \cap \mathfrak{g}_{1}$. In order to prove the converse, assume $X=X_{1}+X_{0}+X_{-1}$ is the decomposition of $X \in \mathfrak{g}_{0}$ into eigenvectors of $\operatorname{ad}\left(H^{\prime}\right)$. Assume that $[X, I] \subset I$; this is equivalent to $\left[X_{1}, I\right] \subset I$. Since, on the other hand, $\left[X_{1}, I\right] \subset \mathfrak{e}_{1} \cap \mathfrak{g}_{1}=V_{1}$, our assumption means that $\left[X_{1}, I\right]=0$. We have also $\left[X_{1}, V_{0}\right] \subset\left[X_{1}, \mathfrak{e}_{2}\right] \subset \mathfrak{e}_{3}=0$. Therefore, in order to show that $X_{1}=0$, all that remains to be proved is that $\left[X_{1}, V_{1}\right]=0$. So let $w \in V_{1}$ and write $w=T\left(e^{+}, e^{-}\right) w=T\left(w, e^{-}\right) e^{+}=$ $\left[T\left(w, e^{-}\right), e^{+}\right]$. Note that $T\left(w, e^{-}\right)=\left[w, e^{-}\right] \in \mathfrak{g}_{0} \cap \mathfrak{h}_{-1}=\mathfrak{g}_{0} \cap \mathfrak{e}_{1}$. Then we have

$$
\left[X_{1}, w\right]=\left[X_{1},\left[T\left(w, e^{+}\right), e^{+}\right]\right]=\left[\left[X_{1}, T\left(w, e^{+}\right)\right], e^{+}\right]+\left[T\left(w, e^{+}\right),\left[X_{1}, e^{+}\right]\right]=0
$$

(the first term is zero since $X_{1}$ and $T\left(w, e^{+}\right)$belong to the abelian Lie algebra $\mathfrak{g}_{0} \cap \mathfrak{h}_{1}$, the second term is zero since $e^{+} \in I=V_{2}$ and $\left[X_{1}, I\right]=0$ by assumption). It follows that $X_{1}=0$, and thus (5.3) and part (1) are proved.

Now we deduce part (2) of the theorem. First of all, note that $\mathfrak{q}$ equals its own normalizer, for, if $X=X_{-2}+\ldots+X_{2}$ normalizes $\mathfrak{q}$, then $\left[X, H^{\prime}\right] \in \mathfrak{q}$ since the grading element belongs 
to $\mathfrak{q}$, and it follows that $2 X_{2}+X_{1} \in \mathfrak{q}$, whence $X_{2}=X_{1}=0$. In a similar way, using that, for $X_{i} \in \mathfrak{e}_{i}, e^{\operatorname{ad}\left(X_{i}\right)} H^{\prime}=H^{\prime}+\left[X_{i}, H^{\prime}\right]=H^{\prime}-i X_{i}$, one sees that the normalizer $N_{G}(\mathfrak{q})$ is the semidirect product of the "unipotent radical" $\exp \left(\mathfrak{e}_{2} \oplus \mathfrak{e}_{1}\right)$ and the "Levi subgroup" $C_{G}\left(H^{\prime}\right)$ (centralizer of the grading element). The Levi subgroup, in turn, is 3-graded with respect to the grading element $E$ and hence is generated by $\exp \left(\mathfrak{e}_{0} \cap \mathfrak{g}_{1}\right), \exp \left(\mathfrak{e}^{0} \cap \mathfrak{g}_{-1}\right)$ and $C_{G}\left(H^{\prime}\right) \cap C_{G}(E)$. Summing up,

$$
N_{G}(\mathfrak{q})=\left\langle\quad \exp \left(\mathfrak{e}_{-2} \oplus \mathfrak{e}_{-1} \oplus \mathfrak{e}_{0} \cap\left(\mathfrak{g}_{1} \oplus \mathfrak{g}_{-1}\right)\right), \quad C_{G}\left(H^{\prime}\right) \cap C_{G}(E) \quad\right\rangle .
$$

On the other hand, one deduces from Part (1) that the right hand side of (5.4) is equal to $G_{\mathcal{I}}$. In fact, since $G_{\mathcal{I}}$ obviously contains the group $G_{I}$ which acts transitively on $\mathcal{I}$, the main point here is to show that for the base point preserving elements we have

$$
\left.G_{\mathcal{I}} \cap \operatorname{Str}\left(V^{+}, V^{-}\right)=\left\langle\quad \exp \left(\mathfrak{g}_{0} \cap \mathfrak{e}_{1}\right), \quad C_{G}\left(H^{\prime}\right)\right) \cap C_{G}(E) \quad\right\rangle .
$$

But this follows from (5.3) since $\mathfrak{g}_{0} \cap \mathfrak{e}_{1}$ is nilpotent and $C_{G}\left(H^{\prime}\right) \cap C_{G}(E)$ is precisely the group preserving both gradings. Summing up, $G_{\mathcal{I}}=N_{G}(\mathfrak{q})$. Finally, (5.4) shows that $N_{G}(\mathfrak{q})$ is also the group stabilizing the minus-filtration of $\mathfrak{e}$, whence the last statement of Part (2).

The theorem shows that the geometry of intrinsic subspaces of Peirce type is isomorphic to some geometry of 5 -filtrations. Of course, one would then like to describe the intrinsic subspace $\mathcal{I}$ directly in terms of filtrations. By analogy with Theorem 3.8, one is lead to conjecture that $\mathcal{I}$ is the set of all 3 -filtrations which satisfy certain inclusion relations with respect to a fixed 5 -filtration. More precisely:

5.10. Conjecture. With notation being as in Theorem 5.8, the intrinsic subspace $\mathcal{I}$ has the following explicit description in terms of 5-filtrations: if $\mathfrak{f}=\left(\mathfrak{f}_{2} \subset \mathfrak{f}_{1} \subset \mathfrak{f}_{0} \subset \mathfrak{f}_{-1} \subset \mathfrak{g}\right)$ is the minus-filtration belonging to the 5-grading from Theorem 5.8, then $\mathcal{I}$ is the space of all 3 -filtrations that are "squeezed" by $\mathfrak{f}$ in the following sense:

$$
\mathcal{I}=\left\{\mathfrak{a} \in \mathcal{X}^{+} \mid \mathfrak{f}_{2} \subset \mathfrak{a}_{1} \subset \mathfrak{f}_{0}, \mathfrak{f}_{1} \subset \mathfrak{a}_{0} \subset \mathfrak{f}_{-1}\right\}
$$

As for the proof of Theorem 3.8, the proof of the inclusion " $\subset$ " is rather elementary, whereas the proof of the other inclusion involves a certain transitivity result which seems to be related to the problems mentioned at the end of the introduction and will be taken up elsewhere.

5.11. The linear picture. As mentioned in Section 5.7, the space of all inner ideals of $V^{+}$ can be seen as a sort of "linear picture" of the geometry of all intrinsic subspaces of $\mathcal{X}^{+}$. If we restrict our attention to inner ideals $I$ of the type $I=V_{2}$ for some idempotent $\left(e^{+}, e^{-}\right)$, this leads us back to a 3 -graded geometry because the Lie algebra $\mathfrak{g}_{0}$ is 3 -graded: with the notation from Lemma 5.9,

$$
\mathfrak{g}_{0}=\left(\mathfrak{g}_{0} \cap \mathfrak{h}_{1}\right) \oplus\left(\mathfrak{g}_{0} \cap \mathfrak{h}_{0}\right) \oplus\left(\mathfrak{g}_{0} \cap \mathfrak{h}_{-1}\right),
$$

and hence $\left(W^{+}, W^{-}\right):=\left(\mathfrak{g}_{0} \cap \mathfrak{h}_{1}, \mathfrak{g}_{0} \cap \mathfrak{h}_{-1}\right)$ is a Jordan pair; let us call it the small Jordan pair associated to an idempotent. The linear picture of inner ideals conjugate to $I$ is the affine pair geometry corresponding to the small Jordan pair.

For the case of certain Banach Jordan triples, this space is studied in the paper [Ka01] by W. Kaup; more precisely, Kaup considers the space of complemented principal inner ideals I in a $J B^{*}$-triple (which are all Peirce 2 -spaces for a suitable idempotent, cf. loc. cit., Lemma 3.2). The principal result in [Ka01] is that this space carries a natural structure of a complex symmetric Banach manifold (loc. cit., Th. 4.4), and that this Banach manifold has as tangent geometry the Peirce 1-space $V_{1}$. (The last fact indicates that there is a particular relation between the small Jordan pair and the Jordan pair $\left(V_{1}^{+}, V_{1}^{-}\right)$; we do not know if this is a general feature or if it 
is due to the particular assumptions in [Ka01].) Explicit descriptions are given for the case of Cartan factors: in case of the series I, II, III, these spaces are again Grassmannians or products of Grassmannians. The additional symmetric space structure from [Ka01] (which does not appear in our work) comes from the fact that there Jordan triple systems (i.e., 3-graded Lie algebras with involution, or polar geometries in the terminology of [Be02]) are considered, whereas we work with Jordan pairs, i.e., projectively.

\section{Appendix A: Inner ideals in matrix spaces}

A.1. Left- and right ideals. Recall that all left ideals in the associative matrix algebra $A=M(n, n ; \mathbb{K})=\operatorname{End}(\mathfrak{m})\left(\right.$ where $\left.\mathfrak{m}=\mathbb{K}^{n}\right)$ over a field $\mathbb{K}$ are of the form

$$
I=I_{\mathfrak{e}}=\{f \in \operatorname{End}(\mathfrak{m}) \mid \mathfrak{e} \subset \operatorname{ker}(f)\}
$$

for some subspace $\mathfrak{e} \subset \mathfrak{m}$. (In fact, if $I$ is generated by a single element $g$, then $I=A g$, and $f \in A g$ if and only if $\operatorname{ker}(g) \subset \operatorname{ker}(f)$; using the relation $I_{\mathfrak{e}_{1}}+I_{\mathfrak{e}_{2}}=I_{\mathfrak{e}_{1} \cap \mathfrak{e}_{2}}$ and fixing some $\mathbb{K}$ basis $g_{1}, \ldots, g_{r}$ of $I$, it follows then by induction that $I=I_{\cap_{i=1}^{r}} \operatorname{ker}\left(g_{i}\right)$.) In particular, every left ideal is principal, i.e., generated by a single element. By taking transposed matrices, it follows then that all right ideals in $M(n, n ; \mathbb{K})$ are of the form

$$
J=J_{\mathfrak{h}}=\{f \in \operatorname{End}(\mathfrak{m}) \mid \operatorname{im}(f) \subset \mathfrak{h}\}
$$

for some subspace $\mathfrak{h} \subset \mathfrak{m}$. If $\mathbb{K}$ is merely a ring, then it is in general no longer true that all left ideals are of the $\mathbb{K}$-algebra $A$ are of the form $I_{\mathfrak{e}}$.

A.2. Inner ideals in associative algebras. If $A$ is an associative $\mathbb{K}$-algebra, we define a trilinear map $T: A \times A \times A \rightarrow A$ by $T(x, y, z)=x y z+z y x$ and say that a linear subspace $I \subset A$ is an inner ideal if it is stable under multiplication by $A$ "from the inside": $T(I, A, I) \subset I$. Since $T$ is symmetric in $x$ and $z$, this is equivalent to the condition $Q(I) A \subset I$, where the quadratic map is defined by

$$
Q(x) y:=\frac{1}{2} T(x, y, x)=x y x
$$

(by polarization, $T$ may be recovered from $Q$ via $T(x, \cdot, z)=Q(x+z)-Q(x)-Q(z)$ ).

If $I$ is a left ideal of the associative algebra $A$, then $Q(I) A \subset I A I \subset I I \subset I$, and hence $I$ is an inner ideal. Similarly, right ideals are inner. Since obviously the intersection of inner ideals is again an inner ideal, all intersections of left and right ideals are inner ideals. In particular, if $A=\operatorname{End}_{\mathbb{K}}(\mathfrak{m})$ is as above, all subspaces of the form

$$
I_{\mathfrak{e}, \mathfrak{h}}:=I_{\mathfrak{e}} \cap J_{\mathfrak{h}}=\{f \in \operatorname{End}(\mathfrak{m}) \mid \mathfrak{e} \subset \operatorname{ker}(f), \operatorname{im}(f) \subset \mathfrak{h}\}
$$

are inner ideals.

\section{A.3. Inner ideals in spaces of rectangular matrices. Let}

$$
V^{+}:=M(p, q ; \mathbb{K})=\operatorname{Hom}(\mathfrak{m}, \mathfrak{n}), \quad V^{-}:=M(q, p ; \mathbb{K})=\operatorname{Hom}(\mathfrak{n}, \mathfrak{m})
$$

be spaces of rectangular matrices over $\mathbb{K}$, with $\mathfrak{m}=\mathbb{K}^{q}, \mathfrak{n}=\mathbb{K}^{p}$. We define trilinear maps $T^{ \pm}: V^{ \pm} \times V^{\mp} \times V^{ \pm} \rightarrow V^{ \pm}$by $T^{ \pm}(x, y, z)=x y z+z y x$ and quadratic maps $Q^{ \pm}: V^{ \pm} \rightarrow$ $\operatorname{Hom}\left(V^{\mp}, V^{ \pm}\right)$by $Q^{ \pm}(x) y=\frac{1}{2} T^{ \pm}(x, y, x)=x y x$. An inner ideal in $V^{+}$is a linear subspace $I \subset V^{+}$such that $Q^{+}(I) A^{-} \subset A^{+}$. As above, it is seen that subspaces of the form $I_{\mathfrak{e}, \mathfrak{h}}$ with $\mathfrak{e} \subset \mathfrak{m}, \mathfrak{h} \subset \mathfrak{n}$, are inner ideals. 
Proposition A.4. Assume $\mathbb{K}$ is a field (which, by our permanent assumption, is of characteristic different from 2). Then the inner ideals of $V^{+}=\operatorname{Hom}(\mathfrak{m}, \mathfrak{n})$ are precisely the sets $I_{\mathfrak{e}, \mathfrak{h}}=\{f \in \operatorname{Hom}(\mathfrak{m}, \mathfrak{n}) \mid \mathfrak{e} \subset \operatorname{ker} f, \operatorname{im} f \subset \mathfrak{h}\}$ for fixed subspaces $\mathfrak{e} \subset \mathfrak{m}, \mathfrak{h} \subset \mathfrak{n}$. In other words, with respect to suitable bases in $\mathfrak{m}$ and $\mathfrak{n}$, every inner ideal is realized by a space of $p \times q$-matrices of the form

$$
\left(\begin{array}{ll}
A & 0 \\
0 & 0
\end{array}\right)
$$

where $A$ runs through all $r \times s$-matrices, for some fixed $r \leq q$ and $s \leq p$. Such an ideal is principal if and and only if $\operatorname{dim} E+\operatorname{dim} F=\operatorname{dim} \mathfrak{m}$, i.e., $r=s$.

Proof. This result is well-known in the context of Jordan-theory (see, e.g., introduction of [Ka01]). However, we do not know a reference where this result is proved by means of elementary linear algebra only; for the reader who is not specialist in Jordan theory, let us give such a proof here.

We have already remarked that $I_{\mathfrak{e}, \mathfrak{h}}$ is indeed an inner ideal. In order to prove that, conversely, all inner ideals are of this form, let us first determine all principal inner ideals $(g)$, where $g \in \operatorname{Hom}(\mathfrak{m}, \mathfrak{n})$. Since $\mathbb{K}$ is assumed to be a field, we may choose bases such that $g$ is realized by a matrix of rank $r$ of the form $\left(\begin{array}{cc}\mathbf{1}_{r} & 0 \\ 0 & 0\end{array}\right)$. An arbitrary element $X \in \operatorname{Hom}(\mathfrak{m}, \mathfrak{n})$ is written accordingly $\left(\begin{array}{ll}a & b \\ c & d\end{array}\right)$ where $a$ is an $r \times r$-matrix and the other components are rectangular. Then we have

$$
Q(g) X=g X g=\left(\begin{array}{ll}
\mathbf{1} & 0 \\
0 & 0
\end{array}\right)\left(\begin{array}{ll}
a & b \\
c & d
\end{array}\right)\left(\begin{array}{ll}
\mathbf{1} & 0 \\
0 & 0
\end{array}\right)=\left(\begin{array}{ll}
a & 0 \\
0 & 0
\end{array}\right) .
$$

Thus all such matrices for arbitrary $r \times r$-matrices $a$ do indeed belong to $(g)$, and it follows that $(g)=I_{\operatorname{ker}(g), \operatorname{im}(g)}$. This proves that all principal inner ideals are of the form $I_{\mathfrak{e}, \mathfrak{h}}$ with $\operatorname{dim} \mathfrak{e}+\operatorname{dim} \mathfrak{h}=\operatorname{dim}(\mathfrak{m})$.

If $I$ is an arbitrary inner ideal, choose a $\mathbb{K}$-basis $g_{1}, \ldots, g_{s}$ of $I$; then $I$ is the smallest inner ideal containing the principal ideals $\left(g_{1}\right), \ldots,\left(g_{s}\right)$, and the claim follows by induction from the following statement:

Lemma A.5. Let inner ideals of the form $I_{1}:=I_{\mathfrak{e}_{1}, \mathfrak{f}_{1}}$ and $I_{2}:=I_{\mathfrak{e}_{2}, \mathfrak{f}_{2}}$ be given. Then the smallest inner ideal $I_{1} \vee I_{2}$ containing $I_{1}$ and $I_{2}$ is given by

$$
I_{\mathfrak{e}_{1}, \mathfrak{f}_{1}} \vee I_{\mathfrak{e}_{2}, \mathfrak{f}_{2}}=I_{\mathfrak{e}_{1} \cap \mathfrak{e}_{2}, \mathfrak{f}_{1}+\mathfrak{f}_{2}} .
$$

Moreover, we have $I_{\mathfrak{e}_{1}, \mathfrak{f}_{1}} \vee I_{\mathfrak{e}_{2}, \mathfrak{f}_{2}}=I_{\mathfrak{e}_{1}, \mathfrak{f}_{1}}+I_{\mathfrak{e}_{2}, \mathfrak{f}_{1}}+I_{\mathfrak{e}_{1}, \mathfrak{f}_{2}}+I_{\mathfrak{e}_{2}, \mathfrak{f}_{2}}$.

Proof. We fix a basis of $\mathfrak{f}_{1} \cap \mathfrak{f}_{2}$, complete it to bases of $\mathfrak{f}_{1}$, resp. $\mathfrak{f}_{2}$ and finally to a basis of $\mathfrak{n}$. We order this basis of $\mathfrak{n}$ such that first comes the basis of $\mathfrak{f}_{1}$, then the basis of $\mathfrak{f}_{2}$, with the basis of $\mathfrak{f}_{1} \cap \mathfrak{f}_{2}$ in the middle, and finally the other basis vectors. We do the same thing with respect to $\mathfrak{e}_{1}$ and $\mathfrak{e}_{2}$, but we order the basis vectors the other way round. Then $I_{1}$ is represented by matrices having non-zero entries on the upper left rectangle, and $I_{2}$ by matrices having non-zero entries on a rectangle that possibly intersects the first rectangle on the basis vectors corresponding to $\mathfrak{f}_{1} \cap \mathfrak{f}_{2}$ and $\mathfrak{e}_{1} \cap \mathfrak{e}_{2}$. We may find a quadratic submatrix $g$ in $I_{1}+I_{2}$ having coefficients 1 on the diagonal and 0 else and which is of maximal size with respect to these conditions. The inner ideal condition then implies that all elements of the form $Q(g) X$ with $X \in V^{-}$belong to $I_{1} \vee I_{2}$, and the matrix calculation (A.1) shows that these elements fill the whole space of submatrices of the type of $g$. Iterating this procedure for all possible matrices $g$, we see that the smallest matrix rectangle containing both $I_{1}$ and $I_{2}$ belongs to $I_{1} \vee I_{2}$. This proves the lemma and the proposition. 
Corollary A.6. The rank of a matrix $g \in M(p, q ; \mathbb{K})$ having coefficients in a field $\mathbb{K}$ is equal to the maximum of lengths of chains $0 \subset I_{1} \subset \ldots \subset I_{r}=(g)$ of principal inner ideals contained in the principal ideal $(g)$.

Proof. As above, represent $g$ by the matrix $\left(\begin{array}{cc}\mathbf{1}_{r} & 0 \\ 0 & 0\end{array}\right)$. Obviously,

$$
0 \subset\left(\left(\begin{array}{cc}
\mathbf{1}_{1} & 0 \\
0 & 0
\end{array}\right)\right) \subset \ldots \subset\left(\left(\begin{array}{cc}
\mathbf{1}_{r} & 0 \\
0 & 0
\end{array}\right)\right)=(g)
$$

is a chain of inner ideals, and Prop. A.4 shows that it has maximal length. Its length is $r$, which is the matrix rank of $g$.

A.7. Idempotents. Let $\left(V^{+}, V^{-}\right)$be a pair of matrix spaces as above. A pair $\left(e^{+}, e^{-}\right) \in$ $V^{+} \times V^{-}$is called an idempotent if

$$
Q\left(e^{+}\right) e^{-}=e^{+}, Q\left(e^{-}\right) e^{+}=e^{-}, \quad \text { i.e. } \quad e^{+} e^{-} e^{+}=e^{+} \text {, and } e^{-} e^{+} e^{-}=e^{-} .
$$

Lemma A.8. A pair $\left(e^{+}, e^{-}\right) \in \operatorname{Hom}(\mathfrak{m}, \mathfrak{n}) \times \operatorname{Hom}(\mathfrak{n}, \mathfrak{m})$ is an idempotent if and only if there are bases in $\mathfrak{m}$ and $\mathfrak{n}$ such that $e^{+}$and $e^{-}$are simultaneously represented by $p \times q$, resp. $q \times p$ matrices of the form

$$
E^{+}=\left(\begin{array}{cc}
\mathbf{1}_{r} & 0 \\
0 & 0
\end{array}\right), \quad E^{-}=\left(\begin{array}{cc}
\mathbf{1}_{r} & 0 \\
0 & 0
\end{array}\right) .
$$

In particular, every element $x \in \operatorname{Hom}(\mathfrak{m}, \mathfrak{n})$ can be completed to an idempotent $\left(e^{+}, e^{-}\right)=(x, y)$.

Proof. It is clear that the pair of matrices $\left(E^{+}, E^{-}\right)$satisfies the conditions of an idempotent. Conversely, assume $\left(e^{+}, e^{-}\right)$is an idempotent. Decomposing $x \in \mathfrak{m}$ in the form $x=e^{-}\left(e^{+}(x)\right)+$ $\left(x-e^{-}\left(e^{+}(x)\right)\right)$, we see that $\mathfrak{m}=\operatorname{im}\left(e^{-}\right) \oplus \operatorname{ker}\left(e^{+}\right)$, and similarly $\mathfrak{n}=\operatorname{im}\left(e^{+}\right) \oplus \operatorname{ker}\left(e^{-}\right)$. It follows that $\operatorname{im}\left(e^{-}\right) \rightarrow \operatorname{im}\left(e^{+}\right), x \mapsto e^{+}(x)$ is a bijection with inverse induced by $e^{-}$. This map is represented by the identity matrix $\mathbf{1}_{r}$ with respect to suitable bases; completing bases by vectors from the respective kernels leads to the matrix representation $\left(E^{+}, E^{-}\right)$.

For the proof of the last statement, represent $x \in \operatorname{Hom}(\mathfrak{m}, \mathfrak{n})$ by the matrix $E^{+}$and let $y \in \operatorname{Hom}(\mathfrak{n}, \mathfrak{m})$ be an element represented by the matrix $E^{-}$with respect to the same bases.

Proposition A.9 (Peirce decomposition). If $\left(e^{+}, e^{-}\right)$is an idempotent in $V^{+} \times V^{-}=$ $\operatorname{Hom}(\mathfrak{m}, \mathfrak{n}) \times \operatorname{Hom}(\mathfrak{n}, \mathfrak{m})$, then the operator

$$
T\left(e^{+}, e^{-}\right): V^{+} \rightarrow V^{+}, \quad z \mapsto T\left(e^{+}, e^{-}, z\right)
$$

is diagonalizable, with three eigenvalues, namely 0,1 and 2 .

Proof. We represent $\left(e^{+}, e^{-}\right)$by matrices $\left(E^{+}, E^{-}\right)$as above. Then

$$
T\left(E^{+}, E^{-}\right)\left(\begin{array}{ll}
a & b \\
c & d
\end{array}\right)=E^{+} E^{-}\left(\begin{array}{ll}
a & b \\
c & d
\end{array}\right)+\left(\begin{array}{ll}
a & b \\
c & d
\end{array}\right) E^{-} E^{+}=\left(\begin{array}{cc}
2 a & b \\
c & 0
\end{array}\right) .
$$

Hence $T\left(E^{+}, E^{-}\right)$is diagonalizable, with eigenspace decomposition

$$
V^{+}=V_{2} \oplus V_{1} \oplus V_{0}=\left\{\left(\begin{array}{cc}
* & 0 \\
0 & 0
\end{array}\right)\right\} \oplus\left\{\left(\begin{array}{cc}
0 & * \\
* & 0
\end{array}\right)\right\} \oplus\left\{\left(\begin{array}{ll}
0 & 0 \\
0 & *
\end{array}\right)\right\} .
$$

This description shows that $V_{2}$ and $V_{0}$ are inner ideals, according to Section A.3. Moreover, comparison with Prop. A.4 shows: 
Corollary A.10. Every principal inner ideal of $V^{+}$is of the form $V_{2}=V_{2}\left(e^{+}, e^{-}\right)$for some idempotent $\left(e^{+}, e^{-}\right)$.

If, in Lemma A.8, we have $r=\min (\operatorname{dim} \mathfrak{m}, \operatorname{dim} \mathfrak{n})$, then $\left(e^{+}, e^{-}\right)$is a maximal idempotent. In this case, $V_{0}=0$, and then $V_{1}$ is also an inner ideal. If $\operatorname{dim} \mathfrak{m}=\operatorname{dim} \mathfrak{n}$ and $\left(e^{+}, e^{-}\right)$is maximal, then also $V_{1}=0$, i.e. $V=V_{2}$. One sees that, if $\operatorname{dim} \mathfrak{m}=\operatorname{dim} \mathfrak{n}$, the collection of all Peirce 2 -spaces for suitable idempotents is the same as the collection of all Peirce 1-spaces.

A.11. Case of symmetric matrices. Assume $\mathfrak{m}$ is a $\mathbb{K}$ module together with a strongly nondegenerate and symmetric bilinear form $\alpha$, i.e., the map $a: \mathfrak{m} \rightarrow \mathfrak{m}^{*}, x \mapsto \alpha(x, \cdot)$ is bijective. Then, for every linear operator $f: \mathfrak{m} \rightarrow \mathfrak{m}$, the adjoint operator $f^{t}$ is defined as usual. The space $V=\operatorname{Sym}(\mathfrak{m})$ of symmetric operators then defines a pair $\left(V^{+}, V^{-}\right)=(\operatorname{Sym}(\mathfrak{m}), \operatorname{Sym}(\mathfrak{m}))$ which is a sub-pair of the Jordan pair $(\operatorname{Hom}(\mathfrak{m}, \mathfrak{m}),(\operatorname{Hom}(\mathfrak{m}, \mathfrak{m}))$. Moreover, $V$ is then a Jordan algebra over $\mathbb{K}$; but we will not use this fact here. Inner ideals of the pair $\left(V^{+}, V^{-}\right)$are defined as above. The intersection of an inner ideal in the space of all matrices with the space of symmetric matrices is again an inner ideal; this defines inner ideals of the form

$$
\{f \in \operatorname{Sym}(\mathfrak{m}) \mid \mathfrak{e} \subset \operatorname{ker}(f) \operatorname{im}(f) \subset \mathfrak{h}\} .
$$

Since $f$ is symmetric, we have $\operatorname{im}(f)=\operatorname{ker}(f)^{\perp}$, and hence the first condition gives us $\operatorname{im}(f) \subset$ $\mathfrak{e}^{\perp}$. Replacing $\mathfrak{e}$ by $\mathfrak{e} \cap \mathfrak{h}^{\perp}$, we may assume that $\mathfrak{e}=\mathfrak{h}^{\perp}$, and hence the inner ideals in question are all of the form

$$
I_{\mathfrak{e}}=\{f \in \operatorname{Sym}(\mathfrak{o}) \mid \mathfrak{e} \subset \operatorname{ker}(f)\}=\left\{f \in \operatorname{Sym}(\mathfrak{o}) \mid \operatorname{im}(f) \subset \mathfrak{e}^{\perp}\right\},
$$

and it is easily seen (same calculation as in (A.1)) that they are all principal. The proof of the following result is similar to (but more complicated than) the one of Prop. A.4:

Proposition A.12. All inner ideals in the Jordan pair $(\operatorname{Sym}(\mathfrak{m}), \operatorname{Sym}(\mathfrak{m}))$, where $\mathfrak{m}=\mathbb{K}^{n}$ is finite-dimensional over a field $\mathbb{K}$, are principal, and they are all of the form $I_{\mathfrak{e}}$ for a suitable subspace $\mathfrak{e} \subset \mathbb{K}^{n}$.

Idempotents in $(\operatorname{Sym}(n, \mathbb{K}), \operatorname{Sym}(n, \mathbb{K}))$ are defined as above. Similarly as in Lemma A.8, one sees that they are just projectors (i.e., $e^{+}=e^{-}=e$ with $e^{2}=e$ ). In particular, the identity $\mathbf{1}_{n}$ is a maximal idempotent, and as above (case $\operatorname{dim} \mathfrak{m}=\operatorname{dim} \mathfrak{n}$ ), the collection of all $V_{0}$ 's is the same as the one of all $V_{2}$ 's.

\section{Appendix B: Inner ideals in Jordan pairs}

B.1. Linear Jordan pairs. Let $\mathbb{K}$ be a commutative unital ring in which 2 and 3 are invertible. A pair $\left(V^{+}, V^{-}\right)$of $\mathbb{K}$-modules equipped with two trilear maps $T^{ \pm}: V^{ \pm} \times V^{\mp} \times V^{ \pm} \rightarrow V^{ \pm}$is called a (linear) Jordan pair if the following properties are satisfied:

(1) $T^{ \pm}(x, y, z)=T^{ \pm}(z, x, y)$

(2) $T^{ \pm}\left(x, y, T^{ \pm}(u, v, w)\right)=T^{ \pm}\left(T^{ \pm}(x, y, u), v, w\right)-T^{ \pm}\left(u, T^{\mp}(y, x, v), w\right)+T(u, v, T(x, y, w))$

We use the following notation: $T^{ \pm}(x, y, x)=2 Q^{ \pm}(x) y, Q^{ \pm}(x, z)=Q^{ \pm}(x+z)-Q^{ \pm}(x)-Q^{ \pm}(z)=$ $T(x, \cdot, z)$. In any Jordan pair, the fundamental formula

$$
Q(Q(x) y)=Q(x) Q(y) Q(x)
$$


holds ([Lo75, Prop. I.2.2]). For instance, associative algebras or spaces of rectangular matrices with $T^{ \pm}$defined as in Sections A.2 and A.3 are Jordan pairs, and in these cases the fundamental formula is easily checked by a direct calculation.

B.2. Construction of Jordan pairs. Every linear Jordan pair is obtained as follows: assume $\mathfrak{g}=\mathfrak{g}_{1} \oplus \mathfrak{g}_{0} \oplus \mathfrak{g}_{-1}$ is a 3 -graded Lie algebra over $\mathbb{K}$; we may assume that $\mathfrak{g}_{0}=\left[\mathfrak{g}_{1}, \mathfrak{g}_{-1}\right]+\mathbb{K} E$ where $E$ is an Euler operator of the grading (cf. Sections 5.1 and 5.5). Then $\left(V^{+}, V^{-}\right)=\left(\mathfrak{g}_{1}, \mathfrak{g}_{-1}\right)$ with

$$
T^{ \pm}(x, y, z)=-[[x, y], z]
$$

is a linear Jordan pair, and every linear Jordan pair is obtained in this way (this is essentially the so-called "Kantor-Koecher-Tits construction"; cf. [Lo95]; see also [Be00, Section III.3] for an interpretation of this construction in terms of polarized Lie triple systems and their standard inbedding). It is not easy to give a conceptual interpretation of the fundamental formula in this context; on the other hand, it is less difficult to give a conceptual interpretation of the Bergmann operators

$$
B^{ \pm}(x, y):=\operatorname{id}_{V^{ \pm}}-T^{ \pm}(x, y, \cdot)+Q^{ \pm}(x) Q^{\mp}(y) \in \operatorname{End}\left(V^{ \pm}\right)
$$

in the context of 3 -graded Lie algebras (see [BeNe04]). If the pair $(x, y)$ is quasi-invertible (i.e., $B(x, y)$ is invertible), then

$$
\left.\beta(x, y):=\left(B^{+}(x, y), B^{(} y, x\right)^{-1}\right)
$$

belongs to the structure group $\operatorname{Str}\left(V^{+}, V^{-}\right)$which, by definition, is the automorphism group of the Jordan pair $\left(V^{+}, V^{-}\right)$.

B.3. Inner ideals in linear Jordan pairs. A submodule $I \subset V^{+}$is called an inner ideal (in $\left.V^{+}\right)$if $T^{+}\left(I, V^{-}, I\right) \subset I$, or, equivalently, $Q^{+}(I) V^{-} \subset I$. We summarize some general results and notions related to inner ideals (if not otherwise stated, they are quoted from [Lo75]):

(1) For $x \in V^{+},[x]:=Q(x) V^{-}=T\left(x, V^{-}, x\right)$ is an inner ideal, called the principal inner ideal generated by $x$. Similarly, for $(x, y) \in V, B(x, y) V^{+}$is an inner ideal of $V^{+}$. Examples: if $(x, y)=\left(e^{+}, e^{-}\right)$is an idempotent (see below), then $V_{2}=\left[e^{+}\right]$is a principal inner ideal and $V_{0}=B\left(e^{+}, e^{-}\right) V^{+}$also is an inner ideal.

(2) Let $(x):=[x]+\mathbb{K} x$, the inner ideal generated by $x$ (smallest inner ideal containing $x$ ). Then $x \in[x]$ iff $x$ is (von Neumann) regular, i.e., there exists $y \in V^{-}$such that $x=Q(x) y$ (in this case $x$ can be completed to an idempotent $\left(e^{+}, e^{-}\right)=(x, Q(y) x)$, [Lo75, Lemma I.5.2]).

(3) An element $z \in V^{ \pm}$called trivial if $Q(z)=0$. Then $\mathbb{K} x$ is an inner ideal, called a trivial inner ideal. $V$ is called non-degenerate if there are no non-zero trivial elements.

(4) A simple inner ideal $I$ is non-trivial and minimal among non-zero inner ideals of $V^{+}$. Equivalently, $I=V_{2}^{+}(d)$ for a division idempotent $d$; or: $I \neq 0$ and $I=[x]$ for all $0 \neq x \in \mathfrak{m}$ (cf. [Lo89]). An element $x \in V$ is called simple if $(x)$ is a simple inner ideal (iff $x=d_{+}$can be extended to a division idempotent $d=\left(d^{+}, d^{-}\right)$.

(5) descending chain condition (dcc) on a set $M$ of inner ideals: every descending chain $\mathfrak{m}_{1} \supset \mathfrak{m}_{2} \supset \ldots$ of inner ideals $\mathfrak{m}_{i} \in M$ becomes stationary. Similarly: ascending chain condition (acc).

(6) images under structural maps: a structural map between Jordan pairs $V$ and $W$ is a pair of maps $f: V^{+} \rightarrow W^{+}, g: W^{-} \rightarrow V^{-}$such that $T^{+}(f(x), y, f(z))=f T^{+}(x, g(y), z)$, $T^{-}(g(a), b, g(c))=g T^{-}(a, g(b), c)$. Then, if $\mathfrak{m} \subset V^{+}$is an inner ideal, then so is $f(\mathfrak{m}) \subset$ $W^{+}$. Example: $W=V^{o p}, f, g=\left(Q^{+}(u), Q^{-}(u)\right)$; this gives the principal inner ideals.

(7) A complement of an inner ideal $I \subset V^{+}$is an inner ideal $J \subset V^{-}$such that $V^{+}=I \oplus \operatorname{ker}(J)$ and $V^{-}=J \oplus \operatorname{ker}(I)$, where the kernel is defined by $\operatorname{ker}(I)=\left\{y \in V^{-} \mid Q^{+}(I) y=0\right\}$, und 
dually for $\operatorname{ker}(J)$ (see [LoNe94]). Note that $\operatorname{ker}(I)$ lives in the space "dual" to $I$, and that it is in general not an inner ideal.

(8) annihilators: for $X \subset V^{-}$the annihilator

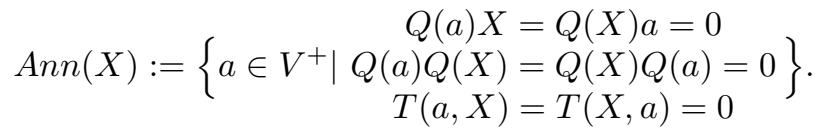

is an inner ideal. If $e$ is an idempotent, then $\operatorname{Ann}\left(e^{-}\right)=V_{0}^{+}(e)$.

B.4. Idempotents. A pair $e=\left(e^{+}, e^{-}\right) \in V^{+} \times V^{-}$such that $Q\left(e^{+}\right) e^{-}=e^{+}, Q\left(e^{-}\right) e^{+}=e^{-}$ is called an idempotent. We summarize some basic results and notions related to idempotents:

(1) Peirce decomposition: the operator $T\left(e^{+}, e^{-}\right)=T\left(e^{+}, e^{-}, \cdot\right)$ is diagonalisable with at most three eigenvalues, $0,1,2$; we write $V=V_{0} \oplus V_{1} \oplus V_{2}$ for the eigenspace decomposition of $V=V^{+}$, and $V_{i}=V_{i}(e)$ is called the Peirce $i$-space of $e$.

(2) Two idempotents $e=\left(e^{+}, e^{-}\right), f=\left(f^{+}, f^{-}\right)$called orthogonal if $Q\left(e^{+}\right) f^{-}=0$ and $Q\left(e^{-}\right) f^{+}=0$. Then $e+f=\left(e^{+}+f^{+}, e^{-}+f^{-}\right)$is again an idempotent.

(3) An orthogonal system of idempotents is an ordered set of pairwise orthogonal idempotents; it is called maximal if it is not properly contained in any larger system. An idempotent $e=\left(e^{+}, e^{-}\right)$is called maximal if $\{e\}$ is a maximal orthogonal system. (Equivalent: the Peirce space $V_{0}(e)$ contains no non-zero idempotent.)

(4) Idempotent $e$ primitive if it is non-zero and cannot be written as the sum of two orthogonal idempotents.

(5) $e=\left(e^{+}, e^{-}\right)$is called a division idempotent $e$ if $V_{2}(e)=\left(V_{2}^{+}, V_{2}^{-}\right)$is a division Jordan pair. (A Jordan pair is called a division Jordan pair if $V \neq 0$ and every non-zero element element is invertible, where $x \in V^{ \pm}$is called invertible if $Q(x): V^{\mp} \rightarrow V^{ \pm}$is invertible. A Jordan pair is division iff it has non-trivial multiplication and no proper inner ideals.)

(6) $e=\left(e^{+}, e^{-}\right)$is called a local idempotent if $V_{2}(e)$ is a local Jordan par. (A local Jordan pair is Jordan pair such that the non-invertible elements form a proper ideal $N$ of $V$; then $V / N$ is division.)

(7) Chain condition on idempotents (cci): no infinite sets of pairwise orthogonal idempotents (then maximal idempotents exist).

(8) A frame is a maximal orthogonal system of local idempotents.

\section{References}

[Be00] Bertram, W., The geometry of Jordan- and Lie structures, Springer Lecture Notes in Mathematics vol. 1754, Berlin 2000.

[Be02] Bertram, W., "Generalized projective geometries: general theory and equivalence with Jordan structures", Adv. Geom. 2 (2002), 329-369.

[Be04] Bertram, W., "From Linear Algebra via Affine Algebra to Projective Algebra", Linear Algebra and its Applications 378 (2004), 109-134.

[Be06] Bertram, W., Differential Geometry, Lie Groups and Symmetric Spaces over General Base Fields and Rings. Mem. AMS, to appear, arXiv: math.DG/ 0502168 .

[BeNe04] Bertram, W. and K.-H. Neeb, "Projective completions of Jordan pairs. I." J. Algebra 277(2) (2004), $193-225$. 
[BeNe05] Bertram, W. and K.-H. Neeb, "Projective completions of Jordan pairs. II" Geom. Dedicata 112 (2005), 73 - 113.

[Bue95] Buekenhout, F. (editor), Handbook of Incidence Geometry, Elsevier, Amsterdam 1995.

[F73] Faulkner, J.R., "On the Geometry of Inner Ideals", J. of Algebra 26 (1973), 1 -9 .

[F80] Faulkner, J.R., "Incidence Geometry of Lie Groups in Quantum Theory", Group theoretical methods in physics (Proc. Eigth Internat. Colloq., Kiryat Anavim, 1979) Ann. Israel Phys. Soc., 3, 73 - 79, Hilger, Bristol, 1980.

[GiKa98] Gindikin,S. and S. Kaneyuki, "On the automorphism group of the generalized conformal structure of a symmetric $R$-space", Diff. Geo. Appl. 8 (1998), 21 33.

[Hua45] Hua, L.-K., "Geometries of matrices. I. Generalizations of von Staudt's theorem", Trans. A.M.S. 57 (1945), 441 - 491.

[Ka01] Kaup, W., "On Grassmannians associated with $J B^{*}$-triples", Math. Z. 236, $567-584,2001$.

[Lo75] Loos, O., Jordan pairs, Springer LN 460, New York 1975.

[Lo89] Loos, O., "On the socle of a Jordan pair", Collect. Math. 40 (2) (1989), 109 125 .

[Lo90] Loos, O., "Filtered Jordan Systems", Comm. Alg. 18 (6) (1990), 1899-1924.

[Lo91] Loos, O., "Diagonalization in Jordan Pairs", J. of Algebra 143 (1) (191), 252268.

[Lo95] Loos, O., "Elementary Groups and Stability for Jordan Pairs", K-Theory 9 (1995), 77 - 116.

[LoNe95] Loos, O. and E. Neher, "Complementation of Inner Ideals in Jordan Pairs", J. Alg. 166 (1994), 255 - 295.

[McC04] McCrimmon, K., A Taste of Jordan Algebras, Springer-Verlag, New York 2004.

Harald Löwe

Technische Universität Braunschweig

Institut Computational Mathematics

Pockelsstrasse 14

D - 38106 Braunschweig

e-mail: h.loewe@tu-bs.de
Wolfgang Bertram

Université Henri Poincaré (Nancy I) Institut Elie Cartan

B.P. 239

F - 54506 Vandœuvre-lès-Nancy Cedex

e-mail: bertram@iecn.u-nancy.fr 